\title{
Karl Auinger
}

Free locally inverse *-semigroups

Czechoslovak Mathematical Journal, Vol. 43 (1993), No. 3, 523-545

Persistent URL: http://dml.cz/dmlcz/128418

\section{Terms of use:}

(C) Institute of Mathematics AS CR, 1993

Institute of Mathematics of the Czech Academy of Sciences provides access to digitized documents strictly for personal use. Each copy of any part of this document must contain these Terms of use.

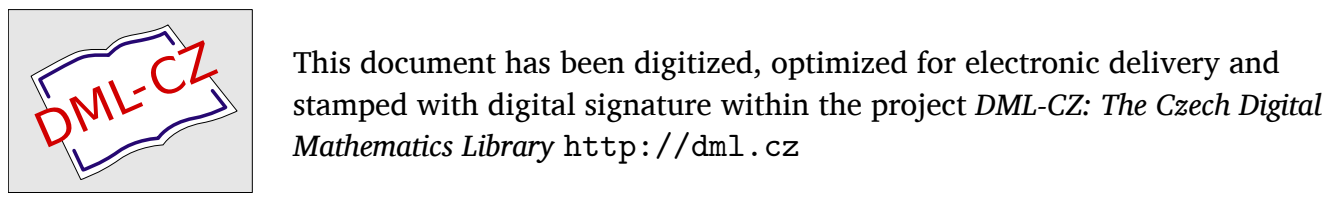




\title{
FREE LOCALLY INVERSE *-SEMIGROUPS
}

\author{
Karl Auinger, Wien
}

(Received January 31, 1992)

\section{INTRODUCTION}

An involution * of a semigroup $S$ is a unary operation $x \mapsto x^{*}$ satisfying

(1) $(x y)^{*}=y^{*} x^{*}$,

(2) $\left(x^{*}\right)^{*}=x$.

The algebra $\left(S, \cdot,^{*}\right)$ is a semigroup with involution or an involutorial semigroup. If in addition

(3) $x x^{*} x=x$

holds then * is a regular involution and the algebra is called a regular *-semigroup. The study of such algebras was suggested by Nordahl and Scheiblich [13] and then conducted by several authors, for instance by Adair [1], Auinger [2, 3], Gerhard and Petrich [5, 6], Nambooripad and Pastijn [12], Petrich [16], Polák [18], Pondělíček [19], Scheiblich [21] and Szendrei [23, 24].

For a class $\mathscr{C}$ of regular semigroups let $\mathscr{C}^{*}$ denote the class of all regular *semigroups $\left(S, \cdot,^{*}\right)$ whose underlying semigroups $(S,$.$) are contained in \mathscr{C}$. The members of $\mathscr{C}^{*}$ will be termed $\mathscr{C}$-*-semigroups (such as completely simple *-semigroups, orthodox *-semigroups etc.). If $\mathscr{C}$ is an $e$-variety (see Hall $[7,8]$ ), that is, if $\mathscr{C}$ is closed under taking direct products, regular subsemigroups and homomorphic inages then $\mathscr{C}^{*}$ forms a variety of algebras of type $\langle 2,1\rangle$. The relatively free objects have been described for several varieties of regular *-semigroups (see $[2,3,5,6,18$, $20,21,23,24])$.

A regular semigroup $S$ is locally inverse if for each idempotent $e$ in $S$, the local submonoid $e S e$ is an inverse semigroup. Locally inverse semigroups have been studied by several authors (see, for instance Pastijn [14, 15] and Nambooripad [11]). It is well known that this class-denoted by $\mathscr{L} \mathscr{S}$-is closed under taking direct products, regular subsemigroups and homomorphic images. Hence the class of all locally inverse 
*-semigroups $\mathscr{L}^{*}$ is a variety. The purpose of the present paper is to describe the free objects in $\mathscr{L}^{*}$. The main result will be an analogue of Scheiblich's well-known description of the free inverse semigroup [20]. Roughly speaking, the completely simple *-semigroups (that is, "*-local groups") play the role for the free locally inverse *-semigroups that groups play for the free inverse semigroups. In fact we shall obtain canonical forms for the elements of the free object $F \mathscr{L}^{*}(X)$, similar to Schein's ones for the inverse case (see [22]). Furthermore, we shall describe $F \mathscr{L}^{*}(X)$ as a certain subsemigroup of a semidirect product of a semilattice by a free completely simple *-semigroup. This will be done in section 4 . In section 2 we shall present some preliminaries, in section 3 some information on free completely simple $*$-semigroups will be given. Finally we shall obtain some further properties of the relatively free objects $F \mathscr{L}^{*}(X)$ in section 5 .

\section{Preliminaries}

For definitions and results concerning semigroups the reader is referred to the books of Howie [9] and Petrich [17] (inverse semigroups).

Let $X$ be any non-empty set, $X^{*}=\left\{x^{*} \mid x \in X\right\}$ be a disjoint copy of $X$ such that $x \mapsto x^{*}$ is a bijection between $X$ and $X^{*}$. Throughout the paper the set $X \cup X^{*}$ will be denoted by $I$. The mapping * $: I \rightarrow I$ then denotes the bijection $x \mapsto x^{*}$, $x^{*} \mapsto x, x \in X$. Let $F^{*}(X)$ be the free semigroup on $I$ which is equipped with the unary operation

$$
{ }^{*}: x_{1} \ldots x_{n} \mapsto x_{n}^{*} \ldots x_{1}^{*} .
$$

We obtain an involutorial semigroup. In fact, $F^{*}(X)$ is the free involutorial semigroup on $X$. By $F^{*}(X)^{1}$ we denote the free involutorial monoid, its identity-the empty word-will be denoted by 1 . Now assume that $X=\left\{z<z^{\prime}<\ldots\right\}$ is well ordered with the least element $z$. Let $I$ be ordered by $z<z^{*}<z^{\prime}<\left(z^{\prime}\right)^{*}<\ldots$. Then $I$ is also well ordered. For each pair $(i, j) \in I \times I$ with $z<i<j$ let $p_{i j}$ be an element not contained in $I$ and such that $p_{i j} \neq p_{k l}$ whenever $(i, j) \neq(k, l)$. Let $P$ denote the set of all these elements. As above, let $P^{*}=\left\{p^{*} \mid p \in P\right\}$ be a disjoint copy of $P, p \mapsto p^{*}$ being a bijection and ${ }^{*}: P \cup P^{*} \rightarrow P \cup P^{*}$ being extended as above. Throughout the paper let $M=P \cup P^{*}$. Also assume that $M \cap I=\emptyset$. Finally we make the convention that for $i>j>z, p_{i j}=p_{j i}^{*}$ and $p_{z i}=p_{i z}=p_{i i}=1$ (denoting the empty word) for all $i \in I$.

In the following we shall introduce three different manipulations of words in $F^{*}(X)$ respectively $F^{*}(X \cup P)^{1}$, two kinds of reductions and one expansion. These operations will be used essentially throughout the paper. First we need some terminology. Let $x_{1} \ldots x_{n} \in F^{*}(Z)$ for any non-empty set $Z$ and $k \in \mathbf{N}$. Then 
$\varrho_{k} x_{1} \ldots x_{n}=x_{1} \ldots x_{\min \{n, k\}}, x_{1} \ldots x_{n} \lambda_{k}=x_{n-\min \{n, k\}+1} \ldots x_{n}, \varrho=\varrho_{1}, \lambda=\lambda_{1}$. Each $\varrho_{k} w$ is an initial segment whereas each $w \lambda_{k}$ is a terminal segment. The length $n$ of the word $w=x_{1} \ldots x_{n}$ will be denoted by $|w|$.

The two mentioned reductions are the following; the first one is the usual reduction of words in the free group.

Definition 1. The mapping $\mathbf{r}: F^{*}(X \cup P)^{1} \rightarrow F^{*}(X \cup P)^{1}$ is defined by $\mathbf{r} 1=1$, $\mathbf{r} y=y$ for all $y \in I \cup M$ where 1 denotes the empty word. Let $n>1$ and suppose that $\mathbf{r} x_{1} \ldots x_{n}=y_{1} \ldots y_{k}(k \geqslant 0)$; then

$$
\mathbf{r} x_{1} \ldots x_{n} x_{n+1}= \begin{cases}y_{1} \ldots y_{k-1} & \text { if } x_{n+1}=y_{k}^{*} \\ y_{1} \ldots y_{k} x_{n+1} & \text { if } x_{n+1} \neq y_{k}^{*}\end{cases}
$$

Here $y_{1} \ldots y_{0}$ stands for the empty word.

Definition 2. The mapping s : $F^{*}(X) \rightarrow F^{*}(X)$ is defined by $\mathbf{s} x=x, \mathbf{s} x y=x y$ for all $x, y \in I$. Let $n>2$ and $\mathrm{s} x_{1} \ldots x_{n}=y_{1} \ldots y_{k}$. Then

$$
\mathbf{s} x_{1} \ldots x_{n} x_{n+1}= \begin{cases}y_{1} \ldots y_{k-1} & \text { if } y_{k-1}=y_{k}^{*}=x_{n+1}, \\ y_{1} \ldots y_{k-2} x_{n+1} & \text { if } y_{k-1}=y_{k}^{*}, y_{k-2}=x_{n+1}^{*}, y_{k-3} \neq y_{k-2}^{*}, \\ y_{1} \ldots y_{k-3} & \text { if } y_{k-1}=y_{k}^{*}, y_{k-2}=x_{n+1}^{*}, y_{k-3}=y_{k-2}^{*} \\ y_{1} \ldots y_{k} x_{n+1} & \text { otherwise. }\end{cases}
$$

Roughly speaking, $\mathbf{r} w$ is obtained by deleting successively each occurrence of some $x^{*} x$ whereas $\mathbf{s} w$ is obtained by successively replacing each occurrence of some $x x^{*} x$ by $x$ and $x y y^{*} x^{*}$ by $x x^{*}(x, y \in I)$. We call $\mathbf{r} w$ the reduced form of $w$ and $\mathrm{s} w$ the weakly reduced form of $w$. The operations $x x^{*} \rightarrow 1$ applied for obtaining $\mathbf{r} w$ are reductions whereas the operations $x x^{*} x \rightarrow x, x y y^{*} x^{*} \rightarrow x x^{*}$ are weak reductions. Further, applying $\mathbf{r}$ respectively $\mathbf{s}$ to subsets $A$ of $F^{*}(X \cup P)^{1}$ or $F^{*}(X)$ means that $\mathbf{r}$ respectively $\mathbf{s}$ will be applied to each element of $A$. It is well-known that the reduced words $\mathbf{r} F^{*}(X \cup P)^{1}$ are canonical forms for the free group on $X \cup P$. If we consider elements of the free group on $X \cup P$, inversion sometimes will be denoted by $^{-1}$ rather than by ${ }^{*}$ and then the words are assumed to be in reduced form. As we shall see in the next section, the weakly reduced words $\mathbf{s} w$ play the role for the free completely simple *-semigroups that reduced words $\mathbf{r} w$ play for the free groups. The third operation on words is the following expansion.

Definition 3. The mapping $\mathbf{e}_{z}: F^{*}(X) \rightarrow F^{*}(X \cup P)^{1}$ is defined by $\mathbf{e}_{z} x=x$ for all $x \in I$ and $\mathbf{e}_{z} x_{1} \ldots x_{n}=x_{1} p_{x_{i}^{*} x_{2}} x_{2} \ldots p_{x_{n-1}^{*} x_{n}} x_{n}$ for all $x_{1} \ldots x_{n} \in F^{*}(X)$, $n>1$. 
Here the index $z$ indicates "normalisation with respect to $z$ ", that is, $p_{z i}=p_{i z}=$ $p_{i i}=1$ for all $i \in I$. As the reductions $\mathbf{s}$ and $\mathbf{r}, \mathbf{e}_{z}$ will be applied to a set $A$ by applying it to each element of $A$.

The following lemma will be used several times without making special mention of. It can be proved easily by induction.

Lemma 2.1. Let $w=x_{1} \ldots x_{n} \in F^{*}(X)$ and $b$ be an initial segment of $\mathbf{s} w$. Then there is an initial segment $\varrho_{k} w$ of $w$ such that $\mathbf{s} \varrho_{k} w=b$.

The semigroups in this paper will be regular *-semigroups (except specially indicated). Hence also "subsemigroups", "homomorphisms", "congruences" etc. are considered to respect multiplication and involution without further making mention of. Similarly, all varieties under study are varieties of algebras of type $\langle 2,1\rangle$. Given such a variety $\mathscr{V}$, the free object in $\mathscr{V}$ on the set $X$ will be denoted by $F^{\mathcal{Y}^{\prime}}(X)$.

\section{COMPLETELY SIMPLE $*$-SEMIGROUPS}

In this section we provide some information on the free completely simple *-semigroup $F \mathscr{C} \mathscr{S}^{*}(X)$. The first lemma has been proved by Petrich [16, Theorem 3.4].

Lemma 3.1. Let $J \neq \emptyset, G$ be a group and $Q=\left(q_{i j}\right)$ be a $J \times J$-matrix with entries in $G$ such that $q_{i j}^{-1}=q_{j i}$ and $q_{i i}=1$ for all $i, j \in J$. Then the Rees matrix semigroup $S=\mathscr{M}(J, G, J ; Q)$, endowed with the usual multiplication and with the involution

$$
(i, g, j)^{*}=\left(j, g^{-1}, i\right)
$$

is a completely simple *-semigroup. Conversely, every completely simple *-semigroup can be so constructed.

The following result is from the same paper ([16, Theorem 4.1]).

Lemma 3.2. A regular *-semigroup $S$ is completely simple if and only if $S$ satisfies the identity $x x^{*}=x y y^{*} x^{*}$.

The free completely simple *-semigroup has been studied by Gerhard and Petrich [6] who obtained a Rees matrix representation of $F \mathscr{C S S}^{*}(X)$ similar to the model of the free completely simple semigroup due to Clifford and Rasin (see [4]). Recently, L. Polák provided a model of $F \mathscr{C O S}^{*}(X)$ by means of canonical forms. In the following, @ES S denotes the fully invariant congruence on $F^{*}(X)$ corresponding to the variety 
$\mathscr{C} \mathscr{S}^{*}$ of all completely simple *-semigroups. The result of Gerhard and Petrich [6, Theorem 7.3] states the following.

Theorem 3.3. Let $X, I=X \cup X^{*}, P$ be as in section 2 and let $G$ denote the free group on $X \cup P$. Then the Rees matrix semigroup $S=\mathscr{M}(I, G, I ; P)$, endowed with the usual multiplication and with the involution of Lemma 3.1 is the free completely simple *-semigroup, freely generated by the set $\left\{\left(x, x, x^{*}\right) \mid x \in X\right\}$.

Theorem 3.3 can be also interpreted in the following way (see also [6, section 8]). Let $G=\mathbf{r} F^{*}(X \cup P)^{1}$ be the set of all reduced words in $F^{*}(X \cup P)^{1}$, endowed the involution of $F^{*}(X \cup P)^{1}$ and the multiplication $w \odot v=\mathbf{r}(w v)$ (in fact, $\mathbf{r} F^{*}(X \cup$ $P)^{1}$ is the free group on $\left.X \cup P\right)$. Then the mapping $\varphi: F^{*}(X) \rightarrow \mathscr{M}(I, G, I ; P)$, defined by $w \varphi=\left(\varrho w, \mathbf{r} \mathbf{e}_{z} w,(w \lambda)^{*}\right)$ is the canonical homomorphism of $F^{*}(X)$ onto $\mathscr{M}(I, G, I ; P) \cong F \mathscr{C} \mathscr{S}^{*}(X)$ which induces the fully invariant congruence $\varrho_{\mathscr{C S}} \cdot$.

On the other hand, L. Polák [18] showed that weak reduction as it is defined in section 2 provides canonical forms for the elements of $F \mathscr{C O S}^{*}(X)$ (this result has been announced at the Conference on Semigroups in Oberwolfach, July 1991). It can be formulated as follows.

Theorem 3.4. Let $\mathbf{s} F^{*}(X)=\left\{\mathbf{s} w \mid w \in F^{*}(X)\right\}$ be the set of all weakly reduced words endowed with the multiplication $w \otimes v=s(w v)$ and with the involution of $F^{*}(X)$. Then the mapping $\mathbf{s}: F^{*}(X) \rightarrow \mathbf{s} F^{*}(X), w \mapsto \mathbf{s} w$ is an epimorphism which induces the fully invariant congruence $\varrho_{\mathscr{C} S}$. In particular, weak reduction provides canonical forms of the elements of $F \mathscr{C S}^{*}(X)$.

Let $\sigma$ denote the equivalence relation on $F^{*}(X)$ defined by $u \sigma v \Leftrightarrow \mathbf{s} u=\mathbf{s} v$. By Lemma 3.2 and Theorem 3.3 it follows immediately that $\sigma \subseteq \varrho_{\mathscr{C} S}$. . The result of L. Polák states that in fact $\sigma=\varrho \mathscr{C S}$. For completeness we shall give an independent proof of this result in the following. Denote by $\varphi: F^{*}(X) \rightarrow \mathscr{M}(I, G, I ; P)$ the canonical homomorphism $w \varphi=\left(\varrho w, \mathbf{r} \mathbf{e}_{z} w,(w \lambda)^{*}\right)$.

Lemma 3.5. Let $w=x_{1} \ldots x_{n} \in F^{*}(X)$ be a word such that $p_{x_{k}^{*} x_{k+1}}=1$ for all $k, 1 \leqslant k<n$ and $w \varphi=\left(x_{1}, 1, x_{1}\right)$; then $\mathrm{s} w=x_{1} x_{1}^{*}$.

Proof. Let $w$ be as above. Immediately we have $x_{n}=x_{1}^{*}$ since $x_{1}=(w \lambda)^{*}$. We show the following. If $w \neq x_{1} x_{1}^{*}$ then $w$ is not weakly reduced. We may assume that $w$ does not contain a subword of the form $x x^{*} x=x, x \in I$. Suppose first that $x_{1} \notin\left\{z, z^{*}\right\}$. The assumptions on $w$ imply that it is a word of the following form

$$
w=x_{1}\left[x_{1}^{*}\right] z w_{0} z^{*} u_{1}\left[u_{1}^{*}\right] z w_{1} z^{*} \ldots u_{k}\left[u_{k}^{*}\right] z w_{k} z^{*}\left[x_{1}\right] x_{1}^{*}
$$


where each $w_{i}$ is a word in the variables $z$ and/or $z^{*}$ or the empty word, $u_{i} \notin\left\{z, z^{*}\right\}$ and the brackets [] indicate that the respective element may or may not occur. If for some $i, u_{i}^{*}$ in the brackets [] actually occurs then $w$ can be weakly reduced. Also, if some $w_{i}$ contains $z$ as well as $z^{*}$ then $z w_{i} z^{*}$ and thus also $w$ can be weakly reduced. Hence we may assume that $w$ is of the following form

$$
w=x_{1}\left[x_{1}^{*}\right] z z_{0} z^{*} u_{1} z z_{1} z^{*} \ldots u_{k} z z_{k} z^{*}\left[x_{1}\right] x_{1}^{*}
$$

where each $z_{i}$ is a power of either $z$ or $z^{*}$ or is the empty word. We know that

$$
1=\mathbf{r} \mathbf{e}_{z} w=\mathbf{r} w=\mathbf{r}\left(x_{1}\left[x_{1}^{*}\right] z_{0} u_{1} z_{1} \ldots u_{k} z_{k}\left[x_{1}\right] x_{1}^{*}\right)
$$

and thus also

$$
\mathbf{r}\left(\left[x_{1}^{*}\right] z_{0} u_{1} z_{1} \ldots u_{k} z_{k}\left[x_{1}\right]\right)=1
$$

Let $u_{0}=\left[x_{1}^{*}\right]$, that is, $u_{0}=x_{1}^{*}$ if $x_{1}^{*}$ actually occurs in the brackets and $u_{0}=1$ otherwise. Similarly let $u_{k+1}=\left[x_{1}\right]$. By relation $(*)$ it follows that there is some $i$ such that $z_{i}=1$ and $u_{i}^{*}=u_{i+1}$. Then $w$ contains a subword of the form $u_{i} z z^{*} u_{i}^{*}$. Hence if $w$ contains some $u_{i}(1 \leqslant i \leqslant k)$ then $w$ can be weakly reduced. We therefore may assume that $w$ has the form

$$
w=x_{1}\left[x_{1}^{*}\right] z z_{0} z^{*}\left[x_{1}\right] x_{1}^{*}
$$

Again using $\mathbf{r} w=\mathbf{r} \mathbf{e}_{z} w=1$ we obtain $z_{0}=1$ and either each or none of the elements in brackets [] occurs. In any case, $w$ can be weakly reduced to $x_{1} x_{1}^{*}$. If $x_{1} \in\left\{z, z^{*}\right\}$ then, as above, we may assume that $w$ is of the form

$$
w=x_{1} w_{0} z^{*} u_{1}\left[u_{1}^{*}\right] \ldots u_{k}\left[u_{k}^{*}\right] z w_{k} x_{1}^{*}
$$

Now we apply the same procedure as for the previous case.

Corollary 3.6. Let $w=x_{1} \ldots x_{n}=\mathrm{s}\left(x_{1} \ldots x_{n}\right) \in \mathrm{s} F^{*}(X)$ be a weakly reduced word. If $p_{x_{k}^{*} x_{k+1}}=1$ for all $k, 1 \leqslant k<n$ and $w \varphi=\left(x_{1}, 1, x_{1}\right)$ then $w=x_{1} x_{1}^{*}$.

Lemma 3.7. If $w=x_{1} \ldots x_{n} \in F^{*}(X)$ is weakly reduced and $w \varphi=\left(x_{1}, 1, x_{1}\right)$ then $w=x_{1} x_{1}^{*}$.

P r o of. Again it is clear that $x_{n}=x_{1}^{*}$. If $p_{x_{k}^{*} x_{k+1}}=1$ for all $k$ then the assertion is proved by Corollary 3.6. Now suppose that there is some $k$ such that $p_{x_{k}^{*} x_{k+1}} \neq 1$. Let $p_{x_{k}^{*} x_{k+1}}=p_{k}$. Since $\mathbf{r}\left(x_{1} p_{1} x_{2} \ldots x_{n-1} p_{n-1} x_{n}\right)=1$ there are $k<l$ such that $1 \neq$ 
$p_{k}=p_{l}^{*}, p_{k+1}=\ldots=p_{l-1}=1$ and $\mathbf{r}\left(x_{k+1} p_{k+1} x_{k+2} \ldots p_{l-1} x_{l}\right)=\mathbf{r}\left(x_{k+1} \ldots x_{l}\right)=1$. Since $p_{x_{k}^{*} x_{k+1}}=p_{k}=p_{l}^{*}=p_{x_{i}^{*} x_{l+1}}^{*}=p_{x_{l+1} x_{i}^{*}}$ we observe that $x_{k}^{*}=x_{l+1}$, that is, $x_{k}=x_{l+1}^{*}$, and $x_{k+1}=x_{l}^{*}$. Since $p_{k+1}=\ldots=p_{l-1}=1$ and $\mathbf{r}\left(x_{k+1} \ldots x_{l}\right)=1$ we have $\left(x_{k+1} \ldots x_{l}\right) \varphi=\left(x_{k+1}, 1, x_{l}^{*}\right)=\left(x_{k+1}, 1, x_{k+1}\right)$. Since $w$ is weakly reduced, the subword $x_{k+1} \ldots x_{l}$ is also weakly reduced so that by Corollary $3.6, x_{k+1} \ldots x_{l}=$ $x_{k+1} x_{k+1}^{*}$ (and thus $l=k+2$ ). But then $w$ contains a subword $x_{k} x_{k+1} x_{k+1}^{*} x_{k}^{*}$ which contradicts the assumption that $w$ is weakly reduced. Therefore, $p_{x_{k}^{*} x_{k+1}} \neq 1$ cannot be true for any $k$ and thus the assertion follows by Corollary 3.6.

Now we are able to obtain the following result.

Corollary 3.8. If $u=\mathbf{s} u=x_{1} \ldots x_{n}$ and $v=\mathbf{s} v=y_{1} \ldots y_{m} \in \mathbf{s} F^{*}(X)$ are weakly reduced words such that $u \varphi=v \varphi$ then $u=v$.

Proof. First, $u \varphi=\left(\varrho u, \mathbf{r} \mathbf{e}_{z} u,(u \lambda)^{*}\right)$ and $v \varphi=\left(\varrho v, \mathbf{r} \mathbf{e}_{z} v,(v \lambda)^{*}\right)$ so that $x_{1}=y_{1}, x_{n}^{*}=y_{m}^{*}$ and $\mathbf{r} \mathbf{e}_{z} u=\mathbf{r}\left(x_{1} p_{x_{1}^{*} x_{2}} x_{2} \ldots x_{n}\right)=\mathbf{r}\left(y_{1} p_{y_{1}^{*} y_{2}} y_{2} \ldots y_{m}\right)=\mathbf{r} \mathbf{e}_{z} v$. Put $w=u v^{*}=x_{1} \ldots x_{n} y_{m}^{*} \ldots y_{1}^{*}$. Notice that $p_{x_{n}^{*} y_{m}^{*}}=1$ since $x_{n}^{*}=y_{m}^{*}$. Hence $\left(\mathbf{e}_{z} u\right)\left(\mathbf{e}_{z} v^{*}\right)=\mathbf{e}_{z} w$. Using $\mathbf{e}_{z} v^{*}=\left(\mathbf{e}_{z} v\right)^{*}$,

$$
\mathbf{r} \mathbf{e}_{z} w=\mathbf{r}\left[\left(\mathbf{e}_{z} u\right)\left(\mathbf{e}_{z} v^{*}\right)\right]=\mathbf{r}\left[\left(\mathbf{e}_{z} u\right)\left(\mathbf{e}_{z} v\right)^{*}\right]=\mathbf{r}\left[\left(\mathbf{r} \mathbf{e}_{z} u\right)\left(\mathbf{r} \mathbf{e}_{z} v\right)^{*}\right]=1
$$

and thus $w \varphi=\left(x_{1}, 1, y_{1}\right)=\left(x_{1}, 1, x_{1}\right)$. By Lemma 3.7 it follows that $\mathbf{s} w=\mathbf{s}\left(u v^{*}\right)=$ $x_{1} x_{1}^{*}$. The weak reduction of $u v^{*}$ to $x_{1} x_{1}^{*}$ necessarily starts with a subword containing a terminal segment of $u$ and an initial segment of $v^{*}$. The first step of weak reduction therefore is one of the following possibilities:

(1) $x_{n-2} x_{n-1} x_{n} y_{m}^{*} \rightarrow x_{n-2} y_{m}^{*}$ where $x_{n-1}=x_{n}^{*}$ and $x_{n-2}=y_{m}$,

(2) $x_{n} y_{m}^{*} y_{m-1}^{*} y_{m-2}^{*} \rightarrow x_{n} y_{m-2}^{*}$ where $y_{m-1}^{*}=y_{m}$ and $x_{n}=y_{m-2}$,

(3) $x_{n-1} x_{n} y_{m}^{*} y_{m-1}^{*} \rightarrow x_{n-1} y_{m-1}^{*}$ where $x_{n-1}=y_{m-1}\left(\right.$ and $\left.x_{n}=y_{m}\right)$,

(4) $x_{n-1} x_{n} y_{m}^{*} \rightarrow y_{m}^{*}=x_{n-1}\left(=x_{n}^{*}\right)$,

(5) $x_{n} y_{m}^{*} y_{m-1}^{*} \rightarrow x_{n}=y_{m-1}^{*}\left(=y_{m}\right)$.

Cases (1) and (2) cannot occur since $x_{n}=y_{m}$ would imply $w \lambda_{3}=x_{n} x_{n}^{*} x_{n}$ or $v \lambda_{3}=\left(\varrho_{3} v^{*}\right)^{*}=y_{m} y_{m}^{*} y_{m}$. In case (3) we immediately observe that $x_{n-1}=y_{m-1}$. In case (4), after the first weak reduction, we get the word $x_{1} \ldots x_{n-1} y_{m-1}^{*} \ldots y_{1}^{*}$. The next weak reduction is of the form either $\left[x_{n-3}\right] x_{n-2} x_{n-1} y_{m-1}^{*} \rightarrow\left[x_{n-3}\right] y_{m-1}^{*}$ or $x_{n-1} y_{m-1}^{*} y_{m-2}^{*}\left[y_{m-3}^{*}\right] \rightarrow x_{n-1}\left[y_{m-3}^{*}\right]$ or $x_{n-2} x_{n-1} y_{m-1}^{*} y_{m-2}^{*} \rightarrow x_{n-2} y_{m-2}^{*}$. (Brackets [ ] indicate that the respective element may or may not be involved.) In the first case, $x_{n-2}=x_{n-1}^{*}=x_{n}$ which is impossible since $x_{1} \ldots x_{n}$ is weakly reduced. In the second case, either $x_{n-1} y_{m-1}^{*} y_{m-2}^{*}=x_{n-1} x_{n-1}^{*} x_{n-1}$ or $y_{m-2}=y_{m-1}^{*}$ and $x_{n-1}=y_{m-3}$. In any case, $y_{m}^{*}=x_{n-1}=x_{n}^{*}$ implies that $y_{m}^{*} y_{m-1}^{*} \ldots y_{1}^{*}$ is not weakly reduced, a contradiction. Therefore only the third case is possible and we 
infer that $x_{n-1}=y_{m-1}$. In case (5), we get $x_{n-1}=y_{m-1}$ in an analogous way. The assertion now follows by induction on $\min \{|u|,|v|\}$.

R e m a r k. In the definition of the weakly reduced word $\mathbf{s} w$ we started the weak reductions on the left hand side of the word $w$ and moved successively to the right in order to avoid ambiguity. Corollary 3.8 now in particular implies that the weak reductions $x x^{*} x \rightarrow x, x y y^{*} x^{*} \rightarrow x x^{*}$ may be executed in any order to obtain $\mathbf{s} w$. We shall use this fact in the sequel without making mention of.

\section{Free locally inverse *-Semigroups}

In this section we first obtain two identities each of which defines the variety $\mathscr{L}_{\mathscr{I}^{*}}$ of all locally inverse *-semigroups (within the variety of all regular *-semigroups). Then we show that each element of $F \mathscr{L}^{*}(X)$ can be written as a product of certain commuting idempotents and a weakly reduced word. Furthermore, we shall show that this rewriting process provides canonical forms for the elements of $F \mathscr{L}^{*}(X)$. This will be done by showing that $F \mathscr{L}^{*}(X)$ can be realized as a subsemigroup of a certain semidirect product of a semilattice by the free completely simple *-semigroup $F \mathscr{C S}^{*}(X)$.

Theorem 4.1. Let $S$ be a regular *-semigroup. Then $S$ is locally inverse if and only if $S$ satisfies either

(4) $x y y^{*} x^{*} x z z^{*} x^{*}=x z z^{*} x^{*} x y y^{*} x^{*}$

or

$\left(4^{\prime}\right)\left(x y x^{*}\right)\left(x y x^{*}\right)^{*}\left(x y x^{*}\right)^{*}\left(x y x^{*}\right)=\left(x y x^{*}\right)^{*}\left(x y x^{*}\right)\left(x y x^{*}\right)\left(x y x^{*}\right)^{*}$.

Proof. Let $e \in E(S)$; then $e \mathscr{D} e e^{*}$ and therefore $e S e$ and $e e^{*} S e e^{*}=e S e^{*}$ are isomorphic as semigroups (via the mapping $x \mapsto x e^{*}$ ). The semigroup $e S e^{*}$ is invariant under the involution so that $e^{*} e^{*}$ is a regular *-semigroup. The identity (4) implies that $e S e^{*}$ satisfies the identity $y y^{*} x x^{*}=x x^{*} y y^{*}$ whereas $\left(4^{\prime}\right)$ implies that $e S e^{*}$ satisfies the identity $x x^{*} x^{*} x=x^{*} x x x^{*}$. In any case, $e S e^{*}$ is an inverse semigroup (see [17, Chap. XII]). Consequently eSe is an inverse semigroup. Conversely, let $S$ be a locally inverse *-semigroup. Then $x S x^{*}=x x^{*} S x x^{*}$ is a regular *-semigroup which in addition is an inverse semigroup. Hence on $x x^{*} S x x^{*}, u \mapsto u^{*}$ is the unique inverse operation. The elements $x y y^{*} x^{*}=(x y)(x y)^{*}$ and $x z z^{*} x^{*}=(x z)(x z)^{*}$ are idempotents in $x x^{*} S x x^{*}$ and therefore commute. In particular, the identity (4) holds in $S$. Similarly, the elements $\left(x y x^{*}\right)\left(x y x^{*}\right)^{*}$ and $\left(x y x^{*}\right)^{*}\left(x y x^{*}\right)$ are idempotents in $x x^{*} S x x^{*}$ and thus commute. This implies the identity $\left(4^{\prime}\right)$. 
Recall that $\mathrm{s}\left(x_{1} \ldots x_{n}\right)$ denotes the weakly reduced word of $x_{1} \ldots x_{n}$. For $w, v \in$ $F^{*}(X)$ the identity $w=v$ holds in $S \in \mathscr{L}^{*}$ if and only if $w f=v f$ for each homomorphism $f: F^{*}(X) \rightarrow S$. The identity $w=v$ holds in $\mathscr{L}^{*}$ if it holds in each member of $\mathscr{L} \mathscr{I}^{*}$. Similarly as for the inverse case (see [17, Chap. VIII]) we have the following rewriting process for locally inverse *-semigroups. (The proof is a natural analogue of the corresponding proof in $[17$, p. 360]). Here equality = stands for equality in a locally inverse *-semigroup $S$, that is, equality in $\mathscr{L}^{*}$.

Theorem 4.2. Let $S$ be a locally inverse *-semigroup and $x_{1}, \ldots, x_{n} \in S$. Then

$$
x_{1} \ldots x_{n}=\prod_{i=1}^{n-1}\left[\mathbf{s}\left(x_{1} \ldots x_{i}\right) \mathbf{s}\left(x_{1} \ldots x_{i}\right)^{*}\right] \mathbf{s}\left(x_{1} \ldots x_{n}\right) .
$$

Proof. Notice that all idempotents $\mathrm{s}\left(x_{1} \ldots x_{k}\right) \mathrm{s}\left(x_{1} \ldots x_{k}\right)^{*}$ commute since they belong to the local inverse submonoid $x_{1} S x_{1}^{*}$ of $S$. The argument is by induction on $n$. For $n=1$ the assertion is trivial. Let $v=x_{1} \ldots x_{n-1}$ and $\mathrm{s} v=y_{1} \ldots y_{k}$. If $\mathbf{s}(v) x_{n} \neq \mathbf{s}\left(v x_{n}\right)$ then either $\mathbf{s} v=y_{1} \ldots y_{k-3} x_{n}^{*} y_{k}^{*} y_{k}$ (that is, $y_{k-1}=y_{k}^{*}$ and $y_{k-2}=x_{n}^{*}$ ) or $\mathbf{s} v=y_{1} \ldots y_{k-2} x_{n} x_{n}^{*}$ (that is, $y_{k-1}=x_{n}$ and $y_{k}=x_{n}^{*}$ ). For the former case we have

$$
\begin{aligned}
\mathbf{s}(v) \mathbf{s}(v)^{*} \mathbf{s}\left(v x_{n}\right) & =\left(y_{1} \ldots y_{k}\right)\left(y_{1} \ldots y_{k}\right)^{*} y_{1} \ldots y_{k-3}\left[x_{n}^{*} x_{n}\right] \\
& =\left(y_{1} \ldots y_{k}\right)\left(y_{1} \ldots y_{k}\right)^{*} y_{1} \ldots y_{k-3} x_{n}^{*} x_{n} \\
& =\left(y_{1} \ldots y_{k-3} x_{n}^{*} y_{k}^{*} y_{k}\right)\left(y_{k}^{*} y_{k} x_{n} y_{k-3}^{*} \ldots y_{1}^{*}\right)\left(y_{1} \ldots y_{k-3} x_{n}^{*} x_{n}\right) \\
& =\left(y_{1} \ldots y_{k-3}\right)\left(x_{n}^{*} y_{k}^{*} y_{k} x_{n}\right)\left(x_{n}^{*} x_{n} y_{k-3}^{*} \ldots y_{1}^{*} y_{1} \ldots y_{k-3} x_{n}^{*} x_{n}\right) \\
& =\left(y_{1} \ldots y_{k-3}\right)\left(x_{n}^{*} x_{n} y_{k-3}^{*} \ldots y_{1}^{*} y_{1} \ldots y_{k-3} x_{n}^{*} x_{n}\right)\left(x_{n}^{*} y_{k}^{*} y_{k} x_{n}\right) \\
& =\left(y_{1} \ldots y_{k-3} x_{n}^{*}\right)\left(x_{n} y_{k-3}^{*} \ldots y_{1}^{*}\right)\left(y_{1} \ldots y_{k-3} x_{n}^{*}\right)\left(y_{k}^{*} y_{k} x_{n}\right) \\
& =\left(y_{1} \ldots y_{k-3} x_{n}^{*}\right) y_{k}^{*} y_{k} x_{n}=(\mathbf{s} v) x_{n} .
\end{aligned}
$$

The notation $\left[x_{n}^{*} x_{n}\right]$ means that $x_{n}^{*} x_{n}$ actually occurs if $y_{k-3} \neq x_{n}$ and is omitted if $y_{k-3}=x_{n}$. For the latter case we have

$$
\begin{aligned}
(\mathbf{s} v)(\mathbf{s} v)^{*} \mathbf{s}\left(v x_{n}\right) & =\mathbf{s}(v) \mathbf{s}(v)^{*} y_{1} \ldots y_{k-2} x_{n} \\
& =\mathbf{s}(v) \mathbf{s}(v)^{*} y_{1} \ldots y_{k-2} x_{n} x_{n}^{*} x_{n} \\
& =\mathbf{s}(v) \mathbf{s}(v)^{*} \mathbf{s}(v) x_{n}=\mathbf{s}(v) x_{n} .
\end{aligned}
$$

Finally, if $\mathbf{s}(v) x_{n}=\mathbf{s}\left(v x_{n}\right)$ then trivially $\mathbf{s}(v) \mathbf{s}(v)^{*} \mathbf{s}\left(v x_{n}\right)=\mathbf{s}(v) x_{n}$. Now let $n>1$ and suppose that the assertion of the Theorem be true for all $n^{\prime}<n$. That is,

$$
x_{1} \ldots x_{n-1}=\prod_{i=1}^{n-2}\left[\mathbf{s}\left(x_{1} \ldots x_{i}\right) \mathbf{s}\left(x_{1} \ldots x_{i}\right)^{*}\right] \mathbf{s}\left(x_{1} \ldots x_{n-1}\right) .
$$


By the above argument,

$$
\mathbf{s}\left(x_{1} \ldots x_{n-1}\right) x_{n}=\mathbf{s}\left(x_{1} \ldots x_{n-1}\right) \mathrm{s}\left(x_{1} \ldots x_{n-1}\right)^{*} \mathrm{~s}\left(x_{1} \ldots x_{n-1} x_{n}\right)
$$

Multiplying (*) by $x_{n}$ on the right then implies the assertion.

Theorem 4.2 provides strong candidates for canonical forms of the elements of $F \mathscr{L}_{\mathscr{I}^{*}}(X)$. One could expect that for two given words $x_{1} \ldots x_{n}, y_{1} \ldots y_{m} \in F^{*}(X)$, the identity $x_{1} \ldots x_{n}=y_{1} \ldots y_{m}$ holds in $F \mathscr{L}^{*}(X)$ if and only if

(1) $\left\{\mathbf{s}\left(x_{1} \ldots x_{i}\right) \mid 1 \leqslant i \leqslant n\right\}=\left\{\mathbf{s}\left(y_{1} \ldots y_{j}\right) \mid 1 \leqslant j \leqslant m\right\}$,

(2) $\mathbf{s}\left(x_{1} \ldots x_{n}\right)=\mathbf{s}\left(y_{1} \ldots y_{m}\right)$.

By Theorem 4.2, (1) and (2) are sufficient in order that $x_{1} \ldots x_{n}=y_{1} \ldots y_{m}$ holds in $\mathscr{L}^{*}$. However, the converse is not true.

Ex a mp le. The identity $x y=x y y^{*} y$ holds in $\mathscr{L}_{\mathscr{I}^{*}}$. Also $\mathrm{s}(x y)=\mathrm{s}\left(x y y^{*} y\right)$. But $\{\mathbf{s}(x), \mathbf{s}(x y)\}=\{x, x y\} \neq\left\{x, x y, x y y^{*}\right\}=\left\{\mathbf{s}(x), \mathbf{s}(x y), \mathbf{s}\left(x y y^{*}\right), \mathbf{s}\left(x y y^{*} y\right)\right\}$.

In the set of weakly reduced initial segments in (1) one has to take into account the element $\mathbf{s}\left(x_{1} \ldots x_{i}\right)$ as well as $\mathbf{s}\left(x_{1} \ldots x_{i} x_{i}^{*}\right)$ for each $i$. For $w=x_{1} \ldots x_{n}$ let $\hat{\mathbf{s}} w=\left\{\mathbf{s}\left(x_{1} \ldots x_{i}\right), \mathbf{s}\left(x_{1} \ldots x_{i} x_{i}^{*}\right) \mid 1 \leqslant i \leqslant n\right\}$. In the following we shall prove that the identity $x_{1} \ldots x_{n}=y_{1} \ldots y_{m}$ holds in $\mathscr{L}^{*}$ if and only if

(1) $\hat{\mathbf{s}}\left(x_{1} \ldots x_{n}\right)=\hat{\mathbf{s}}\left(y_{1} \ldots y_{m}\right)$

(2) $\mathbf{s}\left(x_{1} \ldots x_{n}\right)=\mathbf{s}\left(y_{1} \ldots y_{m}\right)$.

Notice that the product in Theorem 4.2 will not be influenced if the first part is multiplied by all elements of the form $\mathbf{s}\left(x_{1} \ldots x_{i} x_{i}^{*}\right) \mathbf{s}\left(x_{1} \ldots x_{i} x_{i}^{*}\right)^{*}$ since

$$
\mathbf{s}\left(x_{1} \ldots x_{i}\right) \mathbf{s}\left(x_{1} \ldots x_{i}\right)^{*}=\mathbf{s}\left(x_{1} \ldots x_{i} x_{i}^{*}\right) \mathbf{s}\left(x_{1} \ldots x_{i} x_{i}^{*}\right)^{*}
$$

(= denoting equality in $\mathscr{L}_{\mathscr{I}^{*}}$ ) and all such idempotents commute. Next we obtain some auxiliary definitions and results. The purpose is to reconstruct a weakly reduced element $x_{1} \ldots x_{n} \in \mathbf{s} F^{*}(X)$ from $\mathbf{r} \mathbf{e}_{z}\left(x_{1} \ldots x_{n}\right)$. By Corollary 3.8 this will not be completely possible since $x_{1} \ldots x_{n}=\mathrm{s}\left(x_{1} \ldots x_{n}\right)$ is determined by $x_{1}, x_{n}$ and $\mathbf{r} \mathbf{e}_{z}\left(x_{1} \ldots x_{n}\right)$. However, we shall try to obtain as much information as possible. The idea is the following. Let $\mathbf{r e}_{z}\left(x_{1} \ldots x_{n}\right)=q_{1} \ldots q_{k}$ where $q_{i} \in I \cup M$. If $q_{i} \in M$, that is, $q_{i}=p_{u}{ }^{*} v$ for some $u, v \in I$ then $q_{i}$ will be replaced by $u^{*} u v v^{*}$. If $q_{i}=x \in I$ then $x$ will be left unchanged. However, if $x y$ occurs in $\mathbf{r} \mathbf{e}_{z}\left(x_{1} \ldots x_{n}\right)$ and $x^{*} \neq z \neq y, x^{*} \neq y$ then $x y$ has to be replaced by $x z z^{*} y$ rather than by $x y$ since there is no element from $M$ between $x$ and $y$. Formally we proceed as follows. 
Definition 4. Let $q_{1} \ldots q_{k} \in \mathbf{r} F^{*}(X \cup P)^{1}$ and $x \in I$. For $l=0,1, \ldots, k$ let $w_{l}=w_{l}\left(x, q_{1} \ldots q_{k}\right) \in F^{*}(X)$ be defined by induction. First, $w_{0}=x x^{*}$. Suppose that $w_{l} \in F^{*}(X)$ is already defined for some $l \geqslant 0$. Let

$$
w_{l+1}= \begin{cases}w_{l} q_{l+1} & \text { if }\left(q_{l+1} \in I \text { and }\right) w_{l} \lambda=q_{l+1}^{*}, \\ w_{l} z z^{*} q_{l+1} & \text { if } q_{l+1} \in I \text { and } w_{l} \lambda \neq q_{l+1}^{*}, \\ w_{l} u^{*} u v v^{*} & \text { if } q_{l+1}=p_{u^{*} v} \text { and } w_{l} \lambda=u \\ w_{l} z z^{*} u^{*} u v v^{*} & \text { if } q_{l+1}=p_{u^{*} v} \text { and } w_{l} \lambda \neq u\end{cases}
$$

Notice that for the latter two cases, $u^{*} \neq v$ and $u^{*} \neq z \neq v$ since $p_{u^{*} v} \neq 1$. In the following statements let $w_{l}=w_{l}\left(x, q_{1} \ldots q_{k}\right)$.

Lemma 4.3. If $w_{l} \lambda_{3}=z^{*} y y^{*}$ for some $y \in I$ then $w_{l}=w_{1}$ and $x=y^{*}=z^{*}$.

Proof. If $w_{l}$ contains more than two letters then $l>0$. If $w_{l} \lambda_{3}=z^{*} y y^{*}$ then the first case in the definition of $w_{l}$ applies: $w_{l}=w_{l-1} q_{l}$ where $q_{l} \neq 1$ and $q_{l}^{*}=w_{l-1} \lambda$. If $l=1$ then this necessarily implies $w_{0}=q_{l} q_{l}^{*}$. Thus $x=q_{l}$. Then $q_{l} q_{l}^{*} q_{l}=$ $z^{*} y y^{*}$ implies $y^{*}=x=z^{*}$. If $l>1$ then, since $q_{l-1}^{*} \neq q_{l}, w_{l-1}=w_{l-2} u^{*} u v v^{*}$ or $w_{l-1}=w_{l-2} z z^{*} u^{*} u v v^{*}$ and then $w_{l}=w_{l-2} u^{*} u v v^{*} q_{l}$ or $w_{l}=w_{l-2} z z^{*} u^{*} u v v^{*} q_{l}$. Both alternatives are in contradiction to the assumption on $w_{l} \lambda_{3}$ so that $l>1$ is impossible.

Lemma 4.4. If $w_{l} \lambda_{2}=z z^{*}$ then either $l=0$ and $z=x$ or $l=1$ and $z^{*}=x$. Further, $w_{l} \lambda_{4} \neq t t^{*} t t^{*}$ for any $t \in I$.

Proof. If $l=0$ then $w_{0} \lambda_{2}=z z^{*}$ if and only if $z=x$. Suppose that $w_{1} \lambda_{2}=z z^{*}$. Then $w_{1}=w_{0} z^{*}$ and $w_{0}=z^{*} z$. Hence $z^{*}=x$. Let $l>1$ and suppose that $w_{l} \lambda_{2}=z z^{*}$. Then $w_{l}=w_{l-1} z^{*}, q_{l}=z^{*}$ and $w_{l-1} \lambda=z$. But then $q_{l-1}=z$ which is in contradiction to $q_{l}=z^{*}$ since $q_{1} \ldots q_{l}$ is reduced. Hence $w_{l} \lambda_{2} \neq z z^{*}$ whenever $l>1$. The assertion on $w_{l} \lambda_{4}$ is easy to see.

Lemma 4.5. The word $w_{l}$ does not contain a subword of the form $s t^{*} t s^{*}$ for $s \neq t$ nor a subword of the form $s t t^{*} t t^{*} s^{*}$ for any $s, t \in I$.

Proof. We consider the case $s t^{*} t s^{*}, s \neq t$, first. For $l=0,1$ the assertion can be checked easily. Let $l>1$ and assume that the assertion be true for all $l^{\prime}<l$. If $w_{l}=w_{l-1} q_{l}$ then $w_{l-1} \lambda=q_{l}^{*}$ and so the induction hypothesis on $w_{l-1}$ implies the assertion. If $w_{l}=w_{l-1} z z^{*} q_{l}$ then the assertion follows by $w_{l-1} \lambda \neq q_{l}^{*}$, $w_{l-1} \lambda_{3} \neq z^{*} s s^{*}$ (Lemma 4.3) and the induction hypothesis on $w_{l-1}$. Similarly the assertion follows if $w_{l}=w_{l-1} z z^{*} u^{*} u v v^{*}$. If $w_{l}=w_{l-1} u^{*} u v v^{*}$ then $w_{l-1} \lambda=u \neq v^{*}$ and the assertion follows in this case, too. 
Now consider the word $s t t^{*} t t^{*} s^{*}$ for some $s, t \in I$. Again the assertion can be checked directly if $l=0,1$. Let $l>1$ and assume that the assertion be true for all $l^{\prime}<l$. If $w_{l}=w_{l-1} q_{l}$, then $w_{l} \lambda_{6} \neq s t t^{*} t t^{*} s^{*}$ by Lemma 4.4 and thus by the hypothesis of induction on $w_{l-1}$ the assertion follows. If $w_{l}=w_{l-1} z z^{*} q_{l}$ then $\left(w_{l-1} z z^{*} q_{l}\right) \lambda_{6} \neq s t t^{*} t t^{*} s^{*}$ since $w_{l-1} \lambda_{2} \neq z z^{*}$ by Lemma 4.4 and $\left(w_{l-1} z\right) \lambda_{6} \neq$ $s t t^{*} t t^{*} s^{*}$ since $w_{l-1} \lambda_{4} \neq t t^{*} t t^{*}$. Also, $\left(w_{l-1} z z^{*}\right) \lambda_{6}=s t t^{*} t t^{*} s^{*}$ implies $w_{l-1} \lambda_{4}=$ $z z^{*} z z^{*}$ which is impossible. Again by hypothesis on $w_{l-1}$ the assertion follows. Now consider the case $w_{l}=w_{l-1} u^{*} u v v^{*}$, that is, $q_{l}=p_{u^{*} v}$ and $w_{l-1} \lambda=u$. Similarly as above, $\left(w_{l-1} u^{*}\right) \lambda_{6},\left(w_{l-1} u^{*} u\right) \lambda_{6},\left(w_{l-1} u^{*} u v v^{*}\right) \lambda_{6} \neq s t t^{*} t t^{*} s^{*}$. If $\left(w_{l-1} u^{*} u v\right) \lambda_{6}=$ $s^{*} t t^{*} s^{*}$ then $w_{l-1} \lambda_{3}=v^{*} u^{*} u$. It is impossible that $w_{l-1}=w_{l-2} u$, that is, $w_{l-2} \lambda_{2}=$ $v^{*} u^{*}$ and $q_{l-1}=u$ for then $q_{l-2}=u^{*}$, a contradiction. Hence $w_{l-1}=w_{l-2} v v^{*} u^{*} u$ or $w_{l-1}=w_{l-2} z z^{*} v v^{*} u^{*} u$, that is, $q_{l-1}=p_{v u^{*}}$. But this implies $q_{l-1}=q_{l}^{*}$ which is also impossible. Again the assertion follows by hypothesis on $w_{l-1}$. The case $w_{l}=w_{l-1} z z^{*} u^{*} u v v^{*}$ can be treated in an analogous way.

Re mark. Lemma 4.5 in fact assures that $\mathrm{s} w_{l}$ can be obtained by using solely weak reductions of the form $x x^{*} x \rightarrow x, x \in I$.

Corollary 4.6. $\left(\mathrm{s} w_{l}\right) \lambda_{3} \neq z^{*} y y^{*}$ for any $y \in I$.

Proof. This is trivial if $l=0$ and can be checked directly if $l=1$. Let $l>1$. If $w_{l}=w_{l-1} q_{l}$ then $w_{l-1}=w_{l-2} u^{*} u v v^{*}$ or $w_{l-1}=w_{l-2} z z^{*} u^{*} u v v^{*}$ and $q_{l}=v$. The last three letters in the word obtained by the weak reduction $w_{l} \lambda_{3}=v v^{*} v \rightarrow v$ are $u^{*} u v$. By Lemma 4.5 (and the above remark), the element $u$ in $u^{*} u v$ cannot be eliminated by further weak reductions. Since $u^{*} \neq v$ the assertion follows. If $w_{l}=w_{l-1} z z^{*} q_{l}$ we consider two cases. Case (i) $q_{l}=z$. Then $w_{l} \lambda_{4}=s z z^{*} z$ for some $s \neq z^{*}$. Now $w_{l} \lambda_{3}=z z^{*} z$ will be weakly reduced to $z$, but using Lemma 4.5 again, the element $s$ cannot be removed by any further weak reduction. Case (ii) $q_{l} \neq z$. Then $w_{l} \lambda_{2}=z^{*} q_{l}$ and again the letter $z^{*}$ cannot be eliminated by further weak reduction. Finally, if $w_{l}=w_{l-1} u^{*} u v v^{*}$ or $w_{l}=w_{l-1} z z^{*} u^{*} u v v^{*}$ then in both cases $z^{*} \neq u$ and since $u \neq v^{*}$, again by Lemma 4.5 and the remark thereafter, $u$ cannot be removed by weak reduction.

Corollary 4.7. If $\left(\mathrm{s} w_{l}\right) \lambda_{2}=z z^{*}$ then $l=0$ and $x=z$.

Proof. By Lemma 4.5 (and the remark thereafter), $\left(\mathrm{s} w_{l}\right) \lambda_{2}=z z^{*}$ implies $w_{l} \lambda_{2}=z z^{*}$. Hence by Lemma $4.4, l=0$ and $x=z$ or $l=1$. But in the latter case $w_{1}=z^{*} z z^{*}$ and then $\mathrm{s} w_{l}=z^{*}$.

Lemma 4.8. $\mathbf{r} \mathbf{e}_{z} w_{l}\left(x, q_{1} \ldots q_{k}\right)=q_{1} \ldots q_{l}$ and $\mathbf{r} \mathbf{e}_{z} w_{0}=1$. 
Proof. The argument is by induction on $l$. If $l=0$ then this is trivial. Let $l>0$ and suppose the assertion be true for all $l^{\prime}<l$. For the respective cases of Definition 4 we have

$$
w_{l}=\left\{\begin{array}{l}
w_{l-1} q_{l}, \\
w_{l-1} z z^{*} q_{l}, \\
w_{l-1} u^{*} u v v^{*}, \\
w_{l-1} z z^{*} u^{*} u v v^{*}
\end{array} \quad \text { and } \mathbf{e}_{z} w_{l}=\left\{\begin{array}{l}
\left(\mathbf{e}_{z} w_{l-1}\right) q_{l}, \\
\left(\mathbf{e}_{z} w_{l-1}\right) z z^{*} q_{l}, \\
\left(\mathbf{e}_{z} w_{l-1}\right) u^{*} u p_{u^{*} v} v v^{*}, \\
\left(\mathbf{e}_{z} w_{l-1}\right) z z^{*} u^{*} u p_{u^{*} v} v v^{*} .
\end{array}\right.\right.
$$

From this it follows easily that $\mathbf{r} \mathbf{e}_{z} w_{l}=q_{1} \ldots q_{l}$ if $\mathbf{r} \mathbf{e}_{z} w_{l-1}=q_{1} \ldots q_{l-1}$.

Definition 5. Let $x_{1} \ldots x_{n} \in F^{*}(X)$ be a word, let $q_{1} \ldots q_{k}=\mathbf{r} \mathbf{e}_{z}\left(x_{1} \ldots x_{n}\right)=$ $\mathbf{r}\left(x_{1} p_{x_{i}^{*} x_{2}} x_{2} \ldots x_{n}\right)$ and let $w_{k}$ be as in Definition 4. Put

$$
w\left(x_{1}, \mathbf{r} \mathbf{e}_{z}\left(x_{1} \ldots x_{n}\right)\right)=\mathbf{s}\left(w_{k}\left(x_{1}, q_{1} \ldots q_{k}\right)\right) .
$$

Corollary 4.9. Let $x_{1} \ldots x_{n} \in F^{*}(X)$; then

$$
\mathbf{r} \mathbf{e}_{z} w\left(x_{1}, \mathbf{r} \mathbf{e}_{z}\left(x_{1} \ldots x_{n}\right)\right)=\mathbf{r e}_{z}\left(x_{1} \ldots x_{n}\right) .
$$

Proof. If for $a, b \in F^{*}(X), \mathbf{s} a=\mathbf{s} b$ then by Theorem 3.3, $\mathbf{r} \mathbf{e}_{z} a=\mathbf{r} \mathbf{e}_{z} b$. Let $\mathbf{r} \mathbf{e}_{z}\left(x_{1} \ldots x_{n}\right)=q_{1} \ldots q_{k}$. Using Lemma 4.8, we obtain

$$
\begin{aligned}
r \mathbf{e}_{z} w\left(x_{1}, \mathbf{r} \mathbf{e}_{z}\left(x_{1} \ldots x_{n}\right)\right) & =\mathbf{r} \mathbf{e}_{z}\left(\mathbf{s} w_{k}\left(x_{1}, \mathbf{r} \mathbf{e}_{z}\left(x_{1} \ldots x_{n}\right)\right)\right) \\
& =\mathbf{r} \mathbf{e}_{z} w_{k}\left(x_{1}, \mathbf{r} \mathbf{e}_{z}\left(x_{1} \ldots x_{n}\right)\right) \\
& =q_{1} \ldots q_{k}=\mathbf{r} \mathbf{e}_{z}\left(x_{1} \ldots x_{n}\right) .
\end{aligned}
$$

We are able to formulate the following important result.

Theorem 4.10. Let $x_{1} \ldots x_{n}=\mathrm{s}\left(x_{1} \ldots x_{n}\right) \in \mathrm{s} F^{*}(X)$ be a weakly reduced word. Let $w=w\left(x_{1}, \mathbf{r} \mathbf{e}_{z}\left(x_{1} \ldots x_{n}\right)\right)$. Then

$$
x_{1} \ldots x_{n}= \begin{cases}w & \text { iff } w \lambda=x_{n}, \\ w x_{n}^{*} x_{n} & \text { iff } w \lambda=z^{*} \neq x_{n}, \\ w z z^{*} & \text { iff } w \lambda \neq z^{*}=x_{n} \\ w z z^{*} x_{n}^{*} x_{n} & \text { iff } w \lambda \neq z^{*} \neq x_{n}, w \lambda \neq x_{n} .\end{cases}
$$

Pr o of. Denote these four different cases by (1)-(4). Notice that (1)-(4) are pairwise disjoint and each possible case is covered by one of these. In case (1), w is 
clearly weakly reduced. If in case (2) $w x_{n}^{*} x_{n}$ could be weakly reduced then $|w| \geqslant 3$ and $w \lambda_{3}=x_{n} z z^{*}$ which is a contradiction to Corollary 4.7. Hence $w x_{n}^{*} x_{n}$ is weakly reduced. The respective elements of cases (3) and (4) are weakly reduced by Corollary 4.6. Now consider the canonical mapping $\varphi: F^{*}(X) \rightarrow F \mathscr{C} \mathscr{S}^{*}(X)=\mathscr{M}(I, G, I ; P)$ given by $a \mapsto a \varphi=\left(\varrho a, \mathbf{r} \mathbf{e}_{z} a,(a \lambda)^{*}\right)$. Letting $a \in\left\{w, w x_{n}^{*} x_{n}, w z z^{*}, w z z^{*} x_{n}^{*} x_{n}\right\}$ denote any one of the respective cases (1)-(4) then $\left(x_{1} \ldots x_{n}\right) \varphi=a \varphi$. Since $x_{1} \ldots x_{n}$ as well as $a$ is weakly reduced, by Corollary 3.8 we have $x_{1} \ldots x_{n}=a$.

Immediately we have the following result.

Corollary 4.11. Let $x_{1} \ldots x_{n}=\mathbf{s}\left(x_{1} \ldots x_{n}\right) \in \mathbf{s} F^{*}(X)$ be a weakly reduced word and let $w=w\left(x_{1}, \mathbf{r} \mathbf{e}_{z}\left(x_{1} \ldots x_{n}\right)\right)$. If $x_{n-1} \neq x_{n}^{*}$ then $w=x_{1} \ldots x_{n}$.

By Theorem 4.2 we know that for $a, b \in F^{*}(X), a \varrho \mathscr{L} \mathscr{F} \cdot b$ if $(\hat{\mathbf{s}} a, \mathbf{s} a)=(\hat{\mathbf{s}} b, \mathbf{s} b)$. In the following we shall prove the converse. For this purpose we construct a locally inverse *-semigroup in which the identity $a=b$ holds if and only if $(\hat{\mathbf{s}} a, \mathbf{s} a)=(\hat{\mathbf{s}} b, \mathbf{s} b)$. As in section 2 let $G=F \mathscr{G}(X \cup P)$ be the free group on $X \cup P$. In the following, inverses in this group will be indicated by ${ }^{-1}$ rather than by *. In particular, $p_{x^{*} y}=p_{y x^{*}}^{-1}$ for any $x, y \in I$ and we assume that multiplication automatically results in reduced words. Let $Y=F \mathscr{S}(G)$ be the free semilattice generated by $G$. That is, $Y$ consists of all finite non-empty subsets of $G$, endowed with the binary operation of set theoretical union. For $A \in Y, g \in G$ let $g A=\{g a \mid a \in A\}$. According to this definition, the group $G$ acts on the semilattice $Y$ as a group of automorphisms. Now let $S=I \times Y \times G \times I$, endowed with the multiplication

$$
(i, A, g, j)(k, B, h, l)=\left(i, A \cup g p_{j k} B, g p_{j k} h, l\right)
$$

and involution

$$
(i, A, g, j)^{*}=\left(j, g^{-1} A, g^{-1}, i\right) .
$$

By [12, Example 1.7], $S$ is a locally inverse *-semigroup. In fact, $S$ is a perfect rectangular band of $E$-unitary inverse semigroups (see [14]). Let $\chi: F^{*}(X) \rightarrow S$ be the unique extension of the mapping $x \mapsto\left(x,\{1, x\}, x, x^{*}\right), x \in X$, to a homomorphism. Let $x_{1} \ldots x_{n} \in F^{*}(X)$. Using induction, it can be easily seen that

$$
\left(x_{1} \ldots x_{n}\right) \chi=\left(x_{1},\left\{1, x_{1}, x_{1} p_{x_{\mathfrak{i}}^{*} x_{2}}, \ldots, x_{1} p_{x_{\mathfrak{i}}^{*} x_{2}} x_{2} \ldots x_{n}\right\}, x_{1} p_{x_{\mathfrak{i}} x_{2}} x_{2} \ldots x_{n}, x_{n}^{*}\right) .
$$

Since the elements $x_{1} p_{x_{\mathrm{i}} x_{2}} x_{2} \ldots$ are in the group $G$ and

$$
x_{1} p_{x_{1}^{*} x_{2}} x_{2} \ldots p_{x_{k-1}^{*} x_{k}}=x_{1} p_{x_{i}^{*} x_{2}} x_{2} \ldots p_{x_{k-1}^{*} x_{k}} x_{k} x_{k}^{-1},
$$


the homomorphism $\chi$ provides the following information on a given word $a=$ $x_{1} \ldots x_{n} \in F^{*}(x)$ :

(1) $x_{1}$

(2) $\left\{\mathbf{r} \mathbf{e}_{z}\left(x_{1} \ldots x_{i} x_{i}^{*}\right), \mathbf{r} \mathbf{e}_{z}\left(x_{1} \ldots x_{i}\right) \mid 1 \leqslant i \leqslant n\right\}$,

(3) $\mathbf{r} \mathbf{e}_{z}\left(x_{1} \ldots x_{n}\right)$

(4) $x_{n}$.

By Theorem 3.3 and Corollary 3.8 it follows that $\mathbf{s} a$ is uniquely determined by the triple $\left(x_{1}, \mathbf{r} \mathbf{e}_{z} a, x_{n}^{*}\right)$. Theorem 4.10 shows how $\mathbf{s} a$ can be reconstructed from the data $x_{1}, \mathbf{r e}_{z} a$ and $x_{n}$. Further, by Theorem 3.3, the canonical homomorphism $\varphi: F^{*}(X) \rightarrow F \mathscr{C} \mathscr{S}^{*}(X)=\mathscr{M}(I, G, I ; P)$ is given by $a \mapsto\left(\varrho a, \mathbf{r} \mathbf{e}_{z} a,(a \lambda)^{*}\right)$. In particular, $\mathbf{r} \mathbf{e}_{z} a=\mathbf{r} \mathbf{e}_{z} a^{\prime}$ whenever $a \varrho_{\mathscr{C} S *} a^{\prime}$. Consequently, (2) in fact is the following set

$$
\left\{r \mathbf{e}_{z} \mathbf{s}\left(x_{1} \ldots x_{i} x_{i}^{*}\right), \mathbf{r} \mathbf{e}_{z} \mathbf{s}\left(x_{1} \ldots x_{i}\right) \mid 1 \leqslant i \leqslant n\right\} .
$$

For each $i$ let $s_{i}=\mathbf{s}\left(x_{1} \ldots x_{i}\right)$ and $t_{i}=\mathbf{s}\left(x_{1} \ldots x_{i} x_{i}^{*}\right)$. Then either $s_{i} \lambda_{2} \neq\left(s_{i} \lambda\right)^{*}\left(s_{i} \lambda\right)$ or $t_{i} \lambda_{2} \neq\left(t_{i} \lambda\right)^{*}\left(t_{i} \lambda\right)$. Let $u_{i}=w\left(x_{1}, \mathbf{r} \mathbf{e}_{z} s_{i}\right)$ and $v_{i}=w\left(x_{1}, \mathbf{r} \mathbf{e}_{z} t_{i}\right)$ according to Definition 5. Hence by Corollary 4.11 , either $u_{i}=s_{i}$ or $v_{i}=t_{i}$. Also, since $\mathbf{s}\left(\mathbf{s}\left(a_{1} \ldots a_{k}\right) a_{k+1}\right)=\mathbf{s}\left(a_{1} \ldots a_{k} a_{k+1}\right), a_{j} \in I$, we have

$$
t_{i}=\mathbf{s}\left(s_{i}\left(t_{i} \lambda\right)\right)=\mathbf{s}\left(s_{i}\left(s_{i} \lambda\right)^{*}\right)
$$

and

$$
s_{i}=\mathbf{s}\left(t_{i}\left(s_{i} \lambda\right)\right)=\mathbf{s}\left(t_{i}\left(t_{i} \lambda\right)^{*}\right)
$$

In particular,

$$
\left\{s_{i}, t_{i}\right\} \subseteq\left\{u_{i}, \mathbf{s}\left(u_{i}\left(u_{i} \lambda\right)^{*}\right), v_{i}, \mathbf{s}\left(v_{i}\left(v_{i} \lambda\right)^{*}\right)\right\}
$$

for each $i$ and thus

$$
\hat{\mathbf{s}} a \subseteq\left\{u_{i}, v_{i}, \mathbf{s}\left(u_{i}\left(u_{i} \lambda\right)^{*}\right), \mathbf{s}\left(v_{i}\left(v_{i} \lambda\right)^{*}\right) \mid 1 \leqslant i \leqslant n\right\} .
$$

Now take any $\mathbf{s}\left(x_{1} \ldots x_{i}\right)=x_{1} y_{2} \ldots y_{k} x_{i}$ and consider the word $w=w\left(x_{1}, \mathbf{r} \mathbf{e}_{z} s_{i}\right)=$ $w\left(x_{1}, \mathbf{r} \mathbf{e}_{z} x_{1} y_{2} \ldots y_{k} x_{i}\right)$. We apply Theorem 4.10. If $w \lambda=x_{i}$ then $w=s_{i}=$ $\mathbf{s}\left(x_{1} \ldots x_{i}\right)$ and $\mathbf{s}\left(w(w \lambda)^{*}\right)=\mathbf{s}\left(\mathbf{s}\left(x_{1} \ldots x_{i}\right) x_{i}^{*}\right)=\mathbf{s}\left(x_{1} \ldots x_{i} x_{i}^{*}\right)$. If $w \lambda \neq x_{i}$ then either $\mathbf{s}\left(x_{1} \ldots x_{i}\right)=w x_{i}^{*} x_{i}$ or $\mathbf{s}\left(x_{1} \ldots x_{i}\right)=w z z^{*} x_{i}^{*} x_{i}$. In any case, by Lemma 2.1 , $w=\mathbf{s}\left(x_{1} \ldots x_{l}\right)$ for some suitable $l<i$ and thus $w \in \hat{\mathbf{s}} a$. Further, $\mathbf{s}\left(w(w \lambda)^{*}\right)=$ $\mathbf{s}\left(w x_{l}^{*}\right)=\mathbf{s}\left(x_{1} \ldots x_{l} x_{l}^{*}\right) \in \hat{\mathbf{s}} a$. Similarly it can be shown that $w, \mathbf{s}\left(w(w \lambda)^{*}\right) \in \hat{\mathbf{s}} \boldsymbol{a}$ for $w=w\left(x_{1}, \mathbf{r} \mathbf{e}_{z} t_{i}\right)$ for each $i$. Consequently,

$$
\hat{\mathbf{s}} a=\left\{u_{i}, v_{i}, \mathbf{s}\left(u_{i}\left(u_{i} \lambda\right)^{*}\right), \mathbf{s}\left(v_{i}\left(v_{i} \lambda\right)^{*}\right) \mid 1 \leqslant i \leqslant n\right\} .
$$


In fact, the set $\hat{\mathbf{s}} \boldsymbol{a}$ is uniquely determined by the element $x_{1}$ and the set

$$
\mathbf{r} \mathbf{e}_{z} \hat{\mathbf{s}} a=\left\{\mathbf{r} \mathbf{e}_{z} t_{i}, \mathbf{r} \mathbf{e}_{z} s_{i} \mid 1 \leqslant i \leqslant n\right\} .
$$

Summarizing the results we have the following. Let $\varrho_{\mathscr{L} \mathscr{I}}$. denote the fully invariant congruence on $F^{*}(X)$ corresponding to $\mathscr{L}_{\mathscr{I}^{*}}$ and let $\bar{\varrho}_{\mathscr{I}}$ be the least inverse congruence on $F^{*}(X \cup P)^{1}$, that is, the fully invariant congruence corresponding to the variety $\mathscr{I}$ of all inverse semigroups.

Theorem 4.12. Let $a=x_{1} \ldots x_{n}, b=y_{1} \ldots y_{m} \in F^{*}(X)$ be two words. Then the following assertions are equivalent:

(1) $a \varrho \mathscr{L} \mathscr{\mathscr { S }} \cdot b$,

(2) $(\hat{\mathbf{s}} a, \mathbf{s} a)=(\hat{\mathbf{s}} b, \mathbf{s} b)$,

(3) $\varrho a=\varrho b, \mathbf{e}_{z} a \bar{\varrho}_{\mathscr{g}} \mathbf{e}_{z} b, a \lambda=b \lambda$,

(4) $\left(\varrho a, \mathbf{r} \mathbf{e}_{z} \hat{\mathbf{s}} a, \mathbf{r} \mathbf{e}_{z} a,(a \lambda)^{*}\right)=\left(\varrho b, \mathbf{r} \mathbf{e}_{z} \hat{\mathbf{s}} b, \mathbf{r} \mathbf{e}_{z} b,(b \lambda)^{*}\right)$.

Furthermore, the mapping $\chi: F^{*}(X) \rightarrow S=I \times Y \times G \times I$, defined by

$$
a \chi=\left(\varrho a, \mathbf{r} \mathbf{e}_{z} \hat{\mathbf{s}} a, \mathbf{r} \mathbf{e}_{z} a,(a \lambda)^{*}\right)
$$

is a homomorphism which induces $\varrho \mathscr{L} . \mathscr{q}^{*}$. In particular, the (*-)subsemigroup of $S$ which is generated by the set $\left\{\left(x,\{1, x\}, x, x^{*}\right) \mid x \in X\right\}$ is a model of the free locally inverse *-semigroup on $X$. The mapping $\psi:\left(\varrho a, \mathbf{r} \mathbf{e}_{z} \hat{\mathbf{s}} a, \mathbf{r} \mathbf{e}_{z} a,(a \lambda)^{*}\right) \mapsto$ $\left(\varrho a, \mathbf{r e}_{z} a,(a \lambda)^{*}\right)$ is the canonical homomorphism of $F \mathscr{L}^{*}(X)$ onto $F \mathscr{C}^{*}(X)$.

Proof. By Theorem 4.2 we have (2) $\Rightarrow(1)$. Since $\chi: F^{*}(X) \rightarrow S$ is a homomorphism and $S \in \mathscr{L}^{*}$ we have (1) $\Rightarrow(4)$. Since, as shown above, ( $(\hat{\mathbf{s}} a, \mathbf{s} a)$ can be uniquely reconstructed from ( $\left.\varrho a, \mathbf{r} \mathbf{e}_{z} \hat{\mathbf{s}} a, \mathbf{r} \mathbf{e}_{z} a,(a \lambda)^{*}\right)$ the implication (4) $\Rightarrow(2)$ follows. Using the fact that $\mathbf{r e}_{z}=\mathbf{r} \mathbf{e}_{z} \mathbf{s}$ it follows from the well-known description of $\bar{\varrho}_{\mathscr{g}}$ (see [17, Chap. VIII] and [20]) that $\mathbf{e}_{z} a \bar{\varrho}_{\mathscr{g}} \mathbf{e}_{z} b \Leftrightarrow\left(\mathbf{r} \mathbf{e}_{z} \hat{\mathbf{s}} a, \mathbf{r e}_{z} a\right)=\left(\mathbf{r} \mathbf{e}_{z} \hat{\mathbf{s}} b, \mathbf{r} \mathbf{e}_{z} b\right)$, showing the equivalence of (4) and (3). Since (1) $\Leftrightarrow(4)$, the homomorphism $\chi$ : $F^{*}(X) \rightarrow S$ induces the congruence $\varrho_{\mathscr{L} \mathscr{\mathscr { S }}}$ on $F^{*}(X)$. Consequently $F \mathscr{L}_{\mathscr{L}} \mathscr{\mathscr { I }}^{*}(X) \cong$ $F^{*}(X) \chi \subseteq S=I \times Y \times G \times I$ and $F^{*}(X) \chi$ is precisely the (*-)subsemigroup of $S$ which is generated by the set $\left\{\left(x,\{1, x\}, x, x^{*}\right) \mid x \in X\right\}$. Finally, $\varphi=\chi \psi$ where $\varphi$ : $F^{*}(X) \rightarrow F \mathscr{C} \mathscr{S}(X)=\mathscr{M}(I, G, I ; P)$ is the canonical homomrophism. This implies the assertion on $\psi$.

Since $\mathrm{s}\left(x_{1} \ldots x_{i}\right) \mathrm{s}\left(x_{1} \ldots x_{i}\right)^{*} \varrho_{\mathscr{L} \mathscr{G}} \mathrm{s}\left(x_{1} \ldots x_{i} x_{i}^{*}\right) \mathrm{s}\left(x_{1} \ldots x_{i} x_{i}^{*}\right)^{*}$ by Theorem 4.2 we are motivated to define a canonical form of $x_{1} \ldots x_{n} \varrho_{\mathscr{E} g}$. as follows. For $i=$ $1, \ldots, n-1$ put

$$
r_{i}= \begin{cases}s_{i}=\mathrm{s}\left(x_{1} \ldots x_{i}\right) & \text { if } \mathrm{s}\left(x_{1} \ldots x_{i}\right) \lambda_{2} \neq x_{i}^{*} x_{i} \\ t_{i}=\mathrm{s}\left(x_{1} \ldots x_{i} x_{i}^{*}\right) & \text { if } \mathrm{s}\left(x_{1} \ldots x_{i}\right) \lambda_{2}=x_{i}^{*} x_{i}\end{cases}
$$


Then $r_{i} r_{i}^{*} \varrho_{\mathscr{L} \mathscr{I}} s_{i} s_{i}^{*} \varrho_{\mathscr{L} \mathscr{I}} t_{i} t_{i}^{*}$ and $\left(\prod r_{i} r_{i}^{*}\right) \mathrm{s}\left(x_{1} \ldots x_{n}\right) \varrho_{\mathscr{L} \mathscr{J}} \cdot x_{1} \ldots x_{n}$ so that the element $\left(\prod r_{i} r_{i}^{*}\right) \mathbf{s}\left(x_{1} \ldots x_{n}\right)$ can be interpreted as a canonical form of $x_{1} \ldots x_{n}$ in $F \mathscr{L}^{*}(X)$. The product will be taken over the set $\left\{r_{i} \mid 1 \leqslant i \leqslant n\right\}$ rather than $\{i \mid 1 \leqslant i \leqslant n\}$ since several of the elements $r_{i}$ may coincide. All idempotents $r_{i} r_{i}^{*}$ commute.

\section{Some Properties of the Relatively free object $F \mathscr{L}^{*}(X)$}

Concerning the description of $F \mathscr{L} \mathscr{I}^{*}(X)$ as the subsemigroup of the semidirect product $I \times Y \times G \times I$ which is generated by the set $\left\{\left(x,\{1, x\}, x, x^{*}\right) \mid x \in X\right\}$ (Theorem 4.12), the following question arises. Given $(i, A, g, j) \in I \times Y \times G \times I$; is $(i, A, g, j)$ contained in $F^{*}(X) \chi=F \mathscr{L}_{\mathscr{I}^{*}}(X)$ or not? According to Theorem 3.3 and 4.12 , for each $(i, g, j) \in I \times G \times I$ there is some $A \in Y$ such that $(i, A, g, j) \in$ $F \mathscr{L}^{*}(X)$. Hence the question may be formulated as follows. Given $A \in Y$, $(i, g, j) \in I \times G \times I$; is it true or not that $(i, A, g, j) \in F \mathscr{L}^{*}(X)$ ? For given $i \in I$, $g=q_{1} \ldots q_{k} \in G$ (in reduced form) let $w(i, g)=\mathbf{s}\left(w_{k}(i, g)\right)$ where $w_{k}(i, g)$ is as in Definition 4.

Definition 6. Let $i, j \in I, g \in G$. The element $w(i, g, j)$ will be defined by

$$
w(i, g, j)= \begin{cases}w(i, g) & \text { if } w(i, g) \lambda=j^{*} \\ w(i, g) j j^{*} & \text { if } w(i, g) \lambda \neq j^{*} \text { and either } z=j \text { or } w(i, g) \lambda=z^{*} \\ w(i, g) z z^{*} j j^{*} & \text { if } w(i, g) \lambda \neq j^{*} \neq z^{*} \text { and } w(i, g) \lambda \neq z^{*}\end{cases}
$$

By Theorem $4.10, w(i, g, j)$ is the uniquely determined (weakly reduced) word $w \in \mathbf{s} F^{*}(X)$ such that $w \varphi=\left(\varrho w, \mathbf{r} \mathbf{e}_{z} w,(w \lambda)^{*}\right)=(i, g, j)$. Recall that for a given word $a=x_{1} \ldots x_{n} \in F^{*}(X), \hat{\mathbf{s}} a=\left\{\mathbf{s}\left(x_{1} \ldots x_{i}\right), \mathbf{s}\left(x_{1} \ldots x_{i} x_{i}^{*}\right) \mid 1 \leqslant i \leqslant n\right\}$. By Theorem 4.12 we have that $(i, A, g, j) \in F^{*}(X) \chi=F \mathscr{L}^{*}(X)$ if and only if there is some $a=x_{1} \ldots x_{n} \in F^{*}(X)$ such that

(1) $\varrho a=i$,

(2) $\mathbf{r} \mathbf{e}_{z} \hat{\mathbf{s}} a=A$,

(3) $\mathbf{r} \mathbf{e}_{z} a=g$,

(4) $(a \lambda)^{*}=j$.

We formulate the following criterion.

Theorem 5.1. Let $(i, A, g, j) \in I \times Y \times G \times I$. Then $(i, A, g, j) \in F^{*}(X) \chi$ if and only if

(1) $\mathbf{r} \mathbf{e}_{z} \hat{\mathbf{s}} w(i, g, j) \subseteq A$,

(2) $\mathbf{r} \mathbf{e}_{z} \hat{\mathbf{s}} w(i, h) \subseteq A$ for all $h \in A$. 
Proof. Suppose that $(i, A, g, j) \in F^{*}(X) \chi$. Then there is $a=x_{1} \ldots x_{n} \in$ $F^{*}(X)$ such that

$$
(i, A, g, j)=a \chi=\left(\varrho a, \mathbf{r} \mathbf{e}_{z} \hat{\mathbf{s}} a, \mathbf{r} \mathbf{e}_{z} a,(a \lambda)^{*}\right)
$$

Since $a \varphi=\left(\varrho a, \mathbf{r} \mathbf{e}_{z} a,(a \lambda)^{*}\right)=(i, g, j)$ we have $\mathbf{s} a=w(i, g, j)$. Let $b$ be an initial segment of $w(i, g, j)$. Then there is an initial segment $a^{\prime}$ of $a$ such that $\mathbf{s} a^{\prime}=b=\mathbf{s} b$ (Lemma 2.1). Then also

$$
\mathbf{s}\left(a^{\prime}\left(a^{\prime} \lambda\right)^{*}\right)=\mathbf{s}\left(\left(\mathbf{s} a^{\prime}\right)\left(\left(\mathbf{s} a^{\prime}\right) \lambda\right)^{*}\right)=\mathbf{s}\left(b(b \lambda)^{*}\right)=\mathbf{s}\left((\mathbf{s} b)\left((\mathbf{s} b) \lambda^{*}\right)\right) .
$$

Consequently, $\hat{\mathbf{s}} w(i, g, j) \subseteq \hat{\mathbf{s}} a$ and thus $\mathbf{r} \mathbf{e}_{z} \hat{\mathbf{s}} w(i, g, j) \subseteq \mathbf{r} \mathbf{e}_{z} \hat{\mathbf{s}} a=A$ showing (1). (2) will be shown by a similar argument. Let $h \in A$. Then $h \in \mathbf{r} \mathbf{e}_{z} \hat{\mathbf{s}} a$. That is, $h=\mathbf{r} \mathbf{e}_{z} \mathbf{s}\left(x_{1} \ldots x_{l}\right)$ or $h=\mathbf{r} \mathbf{e}_{z} \mathbf{s}\left(x_{1} \ldots x_{l} x_{l}^{*}\right)$ for some $l \leqslant n$. Suppose that $h=\mathbf{r} \mathbf{e}_{z} \mathbf{s}\left(x_{1} \ldots x_{l}\right)$. By Theorem $4.10, w(i, h)$ is an initial segment of $\mathbf{s}\left(x_{1} \ldots x_{l}\right)$ (or coincides with $\mathbf{s}\left(x_{1} \ldots x_{l}\right)$ ). Each initial segment $b=\mathbf{s} b$ of $w(i, h)$ is of the form $b=\mathbf{s}\left(x_{1} \ldots x_{l^{\prime}}\right) \in \hat{\mathbf{s}} a$ for some $l^{\prime} \leqslant l$ (Lemma 2.1). Furthermore, $\mathbf{s}\left(b(b \lambda)^{*}\right)=$ $\mathbf{s}\left(x_{1} \ldots x_{l^{\prime}} x_{l^{\prime}}^{*}\right) \in \hat{\mathbf{s}} a$. In particular, $\hat{\mathbf{s}} w(i, h) \subseteq \hat{\mathbf{s}} a$. If $h=\mathbf{r} \mathbf{e}_{z} \mathbf{s}\left(x_{1} \ldots x_{l} x_{l}^{*}\right)$ then a similar argument applies. In any case we have thus shown the direct part. To prove the converse suppose that (1) and (2) hold for a given $(i, A, g, j) \in I \times Y \times G \times I$. Consider the element

$$
a=\prod_{h \in A}\left[w(i, h) w(i, h)^{*}\right] w(i, g, j)
$$

Notice that all idempotents $w(i, h) w(i, h)^{*}$ commute. It is clear that $\varrho a=\varrho w(i, h)=$ $i$ for each $h \in A, a \lambda=w(i, g, j)=j^{*}$ and $\mathbf{r} \mathbf{e}_{z} a=\mathbf{r} \mathbf{e}_{z} \mathbf{s} a=\mathbf{r} \mathbf{e}_{z} w(i, g, j)=g$. Let the elements of $A$ be indexed in some way: $A=\left\{h_{1}, \ldots, h_{q}\right\}$. Taking into account that $\varrho w\left(i, h_{l}\right)=i=\varrho w(i, g, j)$ for all $h_{l} \in A$ we have the following

$$
a=\left(i a_{11} \ldots a_{1 m_{1}} a_{1 m_{1}}^{*} \ldots a_{11}^{*} i^{*}\right) \ldots\left(i a_{l 1} \ldots a_{l m_{l}} a_{l m_{1}}^{*} \ldots a_{l 1}^{*} i^{*}\right) \ldots i a_{1} \ldots a_{k}
$$

where $w\left(i, h_{l}\right)=i a_{l 1} \ldots a_{l m_{l}}$ and $w(i, g, j)=i a_{1} \ldots a_{k}$. Consider any initial segment $b$ of $a$. Then $\mathbf{s} b$ is one of the following:

$$
\mathrm{s} b \in\left\{i a_{l 1} \ldots a_{l k_{l}}, i a_{l 1} \ldots a_{l k_{l}} a_{l k_{l}}^{*}, i a_{1} \ldots a_{l}\right\}
$$

where $0 \leqslant k_{l} \leqslant m_{l}$ and $0 \leqslant l \leqslant k$ (here $k_{l}=0$ means $\mathbf{s} b=i$ or $\mathbf{s} b=i i^{*}$ and $l=0$ Ineans $\mathbf{s} b=i)$. Consequently, $\mathbf{s}\left(b\left(b \lambda^{*}\right)\right)$ is one of the following:

$$
\mathbf{s}\left(b(b \lambda)^{*}\right) \in\left\{i a_{l 1} \ldots a_{l k_{l}} a_{l k_{l}}^{*}, i a_{l 1} \ldots a_{l k_{l}}, i a_{1} \ldots a_{l} a_{l}^{*}\right\}
$$


(provided the same convention on $k_{l}$ and $l$ ). In any case we have $\mathbf{s} b, \mathbf{s}\left(b(b \lambda)^{*}\right) \in$ $\hat{\mathbf{s}} w\left(i, h_{l}\right)$ for some $h_{l} \in S$ or $\mathbf{s} b, \mathbf{s}\left(b(b \lambda)^{*}\right) \in \hat{\mathbf{s}} w(i, g, j)$. By conditions (1) and (2) it follows that $\mathbf{r} \mathbf{e}_{z} \hat{\mathbf{s}} a \subseteq A$. On the other hand, for $h_{l} \in A$ we have

$$
\mathbf{s} i a_{11} \ldots a_{1 m_{1}} a_{1 m_{1}}^{*} \ldots i^{*} \ldots i a_{l 1} \ldots a_{l 1} \ldots a_{l m_{l}}=i a_{l 1} \ldots a_{l m_{l}}=w\left(i, h_{l}\right) \in \hat{\mathbf{s}} a
$$

By Lemma 4.8 also $\mathbf{r e}_{z} w\left(i, h_{l}\right)=h_{l}$ and thus $h_{l}=\mathbf{r} \mathbf{e}_{z} w\left(i, h_{l}\right) \in \mathbf{r} \mathbf{e}_{z} \hat{\mathbf{s}} a$. The element $h_{l} \in A$ is arbitrarily chosen so that $A \subseteq \mathbf{r} \mathbf{e}_{z} \hat{\mathbf{s}} a$ and thus $A=\mathbf{r} \mathbf{e}_{z} \hat{\mathbf{s}} a$. Summarizing the converse part we have shown that

$$
(i, A, \boldsymbol{g}, j)=\left(\varrho a, \mathbf{r} \mathbf{e}_{z} \hat{\mathbf{s}} \boldsymbol{a}, \mathbf{r} \mathbf{e}_{z} a,(a \lambda)^{*}\right) \in F^{*}(X) \chi=F \mathscr{L} \mathscr{I}^{*}(X)
$$

The next results concern idempotents and the natural partial order in $F^{*}(X) \chi=$ $F \mathscr{L}^{*}(X)$.

Lemma 5.2. Let $(i, A, g, j) \in I \times Y \times G \times I$. Then $(i, A, g, j)^{2}=(i, A, g, j)$ if and only if $g=p_{i j}$.

Proof. We have $(i, A, g, j)(i, A, g, j)=\left(i, A \cup g p_{j i} A, g p_{j i} g, j\right)$. Hence $(i, A, g, j)$ is idempotent if and only if $g=g p_{j i} g$ and $A \cup g p_{j i} A=A$. The first condition is equivalent to $g=p_{j i}^{-1}$ and thus $g=p_{i j}$. Conversely, if $g=p_{i j}$ then immediately $(i, A, g, j) \in E(I \times Y \times G \times I)$.

Corollary 5.3. Let $w=x_{1} \ldots x_{n} \in F^{*}(X)$. Then $w \varrho \mathscr{L} \mathscr{\mathscr { S }} \cdot w^{2}$ (that is, $w$ is an idempotent in $\left.F \mathscr{L}^{*}(X)\right)$ if and only if either $\mathrm{s} w=x_{1} x_{1}^{*} x_{n}^{*} x_{n}$ or $\mathrm{s} w=x_{1} x_{1}^{*}=$ $x_{n}^{*} x_{n}$.

Proof. We have $w \varrho \mathscr{S} \mathscr{g} \cdot w^{2}$ if and only if $w \chi$ is an idempotent in $F^{*}(X) \chi$. That is, $w \chi=\left(i, A, p_{i j}, j\right)$ by Lemma 5.3. The element $\mathbf{s} w$ is uniquely determined by the parameters $i, p_{i j}, j$, namely $\mathrm{s} w=w\left(i, p_{i j}, j\right)$. By Definition 6 ,

$$
w\left(i, p_{i j}, j\right)= \begin{cases}i i^{*}=j j^{*} & \text { if } i=j, \\ i i^{*} j j^{*} & \text { if } i \neq j .\end{cases}
$$

Since $i=\varrho(\mathbf{s} w)=\varrho w=x_{1}$ and $j=((\mathbf{s} w) \lambda)^{*}=(w \lambda)^{*}$ we observe that $\mathbf{s} w=$ $x_{1} x_{1}^{*} x_{n}^{*} x_{n}$ or $\mathbf{s} w=x_{1} x_{1}^{*}=x_{n}^{*} x_{n}$. Conversely, if $\mathbf{s} w=x_{1} x_{1}^{*} x_{n}^{*} x_{n}$ then $w \chi=$ $\left(x_{1}, A, p_{x_{1} x_{n}^{*}}, x_{n}^{*}\right)$ and $w \chi$ is idempotent. Similarly, if $\mathbf{s} w=x_{1} x_{1}^{*}=x_{n}^{*} x_{n}$ then $w \chi=\left(x_{1}, A, 1, x_{1}\right)=\left(x_{n}^{*}, A, 1, x_{n}\right)$ which is idempotent.

The natural partial order on a regular semigroup has been introduced by Nambooripad [11]. A list of equivalent definitions is given by Mitsch [10]. 
Definition 7. Let $S$ be a regular semigroup, $a, b \in S$. Then $a \leqslant b$ if and only if there are idempotents $e, f \in E\left(S^{\prime}\right)$ such that $a=e b=b f$.

Lemma 5.4. Let $S$ be a regular *-semigroup. Then ${ }^{*}: x \mapsto x^{*}$ is an order automorphism of $(S, \leqslant)$.

Proof. Let $a \leqslant b$, that is, $a=e b=b f$ for some $e, f \in E(S)$. Then $a^{*}=b^{*} e^{*}=$ $f^{*} b^{*}$. Since $e^{*}, f^{*} \in E(S), a^{*} \leqslant b^{*}$. Since ${ }^{*}$ is self-inverse the assertion follows.

For locally inverse $*$-semigroups we give a further characterization of $\leqslant$ which is a natural anologon of the well known definition of $\leqslant$ for the inverse case. In [11] Nambooripad has shown that a regular semigroup is locally inverse if and only if $\leqslant$ is compatible with the multiplication.

Proposition 5.5. Let $S$ be a locally inverse *-semigroup. Then $a \leqslant b$ if and only if $a=a a^{*} b=b a^{*} a$.

Proof. If $a \leqslant b$ then $a^{*} \leqslant b^{*}$ by Lemma 5.4. Compatibility of $\leqslant$ implies $a \leqslant a a^{*} b, a \leqslant b a^{*} a, a^{*} \leqslant a^{*} a b^{*}, a^{*} \leqslant b^{*} a a^{*}, a^{*} a \leqslant b^{*} b$ and $a a^{*} \leqslant b b^{*}$. Now $a^{*} \leqslant a^{*} a b^{*}$ implies $a^{*} b \leqslant a^{*} a b^{*} b=a^{*} a$. Hence $a a^{*} b \leqslant a a^{*} a$ so that $a=a a^{*} b$. Similarly, $a^{*} \leqslant b^{*} a a^{*}$ implies $b a^{*} \leqslant b b^{*} a a^{*}=a a^{*}$. Hence $b a^{*} a \leqslant a$ so that $a=b a^{*} a$. The converse is obvious.

R e m a rk. In the same fashion as for the inverse case several equivalent characterizations of $\leqslant$ in a locally inverse $*$-semigroup can be obtained.

Corollary 5.6. Let $(i, A, g, j),(k, B, h, l) \in I \times Y \times G \times I$. Then $(i, A, g, j) \leqslant$ $(k, B, h, l)$ if and only if $(i, g, j)=(k, h, l)$ and $B \subseteq A$.

Proof. A straightforward calculation shows

$$
(i, A, g, j)(i, A, g, j)^{*}(k, B, h, l)=\left(i, A \cup p_{i k} B, p_{i k} h, l\right)
$$

and

$$
(k, B, h, l)(i, A, g, j)^{*}(i, A, g, j)=\left(k, B \cup h p_{l j} g^{-1} A, h p_{l j}, j\right) .
$$

If $(i, g, j)=(k, h, l)$ and $B \subseteq A$ then immediately from Proposition 5.5. $(i, A, g, j) \leqslant$ $(k, B, h, l)$. Conversely suppose $(i, A, g, j) \leqslant(k, B, h, l)$. By Proposition $5.5, l=$ $j, k=i, g=p_{i k} h=h$ and $A=A \cup p_{i k} B=A \cup B$ so that $B \subseteq A$.

Corollary 5.7. Let $u, v \in F^{*}(X)$. Then $u \varrho \mathscr{L} \mathscr{g}_{*} \leqslant v \varrho \mathscr{L} \mathscr{I}_{*}$ if and only if

(1) $\mathbf{s} u=\mathbf{s} v$,

(2) $\hat{\mathbf{s}} u \supseteq \hat{\mathbf{s}} v$. 
Proof. The inequality $u \leqslant v$ holds in $F \mathscr{L}^{*}(X)$ if and only if $u \chi \leqslant v \chi$ in $F^{*}(X) \chi$. Now

$$
u \chi \leqslant v \chi \Leftrightarrow\left(\varrho u, \mathbf{r} \mathbf{e}_{z} u,(u \lambda)^{*}\right)=\left(\varrho v, \mathbf{r} \mathbf{e}_{z} v,(v \lambda)^{*}\right) \text { and } \mathbf{r} \mathbf{e}_{z} \hat{\mathbf{s}} v \subseteq \mathbf{r} \mathbf{e}_{z} \hat{\mathbf{s}} u .
$$

Immediately we thus have that (1) and (2) imply $u \varrho_{\mathscr{L} \mathscr{I}^{*}} \leqslant v \varrho_{\mathscr{L}} \mathscr{F}^{*}$. Suppose conversely that $\left(\varrho u, \mathbf{r} \mathbf{e}_{z} u,(u \lambda)^{*}\right)=\left(\varrho v, \mathbf{r} \mathbf{e}_{z} v,(v \lambda)^{*}\right)$ and $\mathbf{r} \mathbf{e}_{z} \hat{\mathbf{s}} v \subseteq \mathbf{r} \mathbf{e}_{z} \hat{\mathbf{s}} u$. First we have $\mathbf{s} u=w\left(\varrho u, \mathbf{r} \mathbf{e}_{z} u,(u \lambda)^{*}\right)=w\left(\varrho v, \mathbf{r} \mathbf{e}_{z} v,(v \lambda)^{*}\right)=\mathbf{s} v$. By the process which reconstructs $\hat{\mathbf{s}} u$ from $\varrho u$ and $\mathbf{r} \mathbf{e}_{z} \hat{\mathbf{s}} u$ and $\hat{\mathbf{s}} v$ from $\varrho v$ and $\mathbf{r} \mathbf{e}_{z} \hat{\mathbf{s}} v$ (see end of section 4) it follows that $\hat{\mathbf{s}} v \subseteq \hat{\mathbf{s}} u$.

Definition 8. Let $A \subseteq S$ be a subset of a regular semigroup. Then $A \omega=\{x \in$ $S \mid a \leqslant x$ for some $a \in A\}$.

It is well known that the free inverse semigroup $F \mathscr{I}(X)$ is $E$-unitary (see [17]). This is not true for locally inverse *-semigroups as an $E$-unitary regular semigroup must be orthodox. However, for inverse semigroups $S$ the property of being $E$ unitary is equivalent to the property that the idempotents form a closed subset of $S$ under the natural order, that is $E \omega=E$. This seems to be the appropriate analogue for the locally inverse case.

Corollary 5.8. For the free locally inverse *-semigroup $F \mathscr{L}^{*}(X), E \omega=E$.

Proof. Let $\left(i, A, p_{i j}, j\right),(k, B, h, l) \in F^{*}(X) \chi$ such that $\left(i, A, p_{i j}, j\right) \leqslant$ $(k, B, h, l)$. By Corollary $5.6,\left(i, p_{i j}, j\right)=(k, h, l)$. Hence by Lemma $5.2,(k, B, h, l)=$ $\left(i, B, p_{i j}, j\right)$ is an idempotent.

Finally we mention some more properties of the relatively free object $F \mathscr{L}_{\mathscr{I}^{*}}(X)$. By Nordahl and Scheiblich [13], Green's relations $\mathscr{R}$ and $\mathscr{L}$ on a regular $*$-semigroup admit the following description.

Lemma 5.9. Let $S$ be a regular *-semigroup and $a, b \in S$. Then

(1) $a \mathscr{R} b \Leftrightarrow a a^{*}=b b^{*}$,

(2) $a \mathscr{L} b \Leftrightarrow a^{*} a=b^{*} b$.

For two elements of the semidirect product $I \times Y \times G \times I$ this yields the following characterization:

(1) $(i, A, g, j) \mathscr{R}(k, B, h, l) \Leftrightarrow i=k$ and $A=B$,

(2) $(i, A, g, j) \mathscr{L}(k, B, h, l) \Leftrightarrow j=l$ and $g^{-1} A=h^{-1} B$.

Since for each $w \in F^{*}(X),\left(\varrho w, \mathbf{r} \mathbf{e}_{z} \hat{\mathbf{s}} w\right)$ is uniquely determined by $\hat{\mathbf{s}} w$ and conversely, this leads to the following characterization of Green's relations in $F \mathscr{L}^{*}(X)$.

Propostion 5.10. Let $v, w \in F^{*}(X)$. Then

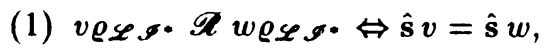

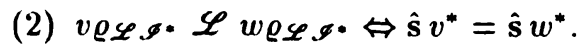


The description of the relation $\mathscr{L}$ also could be formulated directly in terms of $v$ and $w$. However, for this purpose the dual of the operator $\hat{\mathbf{s}}$ is needed. Using a similar idea as in [17, VIII.1.14] the description of $\mathscr{L}$ respectively $\mathscr{P}$ in $I \times Y \times G \times I$ can be used to show that this semidirect product is combinatorial.

Corollary 5.11. $F \mathscr{L}^{*}(X)$ is combinatorial.

Corollary 5.12. $F \mathscr{L}^{*}(X)$ has finite $\mathscr{R}$ - and $\mathscr{L}$-classes. In particular, $F \mathscr{L}^{*}(X)$ is completely semisimple with finite $\mathscr{D}$-classes and is finite- $\mathscr{R}(\mathscr{L}, \mathscr{D})$ above.

Proof. Let $v \in F^{*}(X)$. Then $v \varrho_{\mathscr{L} \mathscr{I}} \mathscr{R}$ is determined by $\hat{\mathbf{s}} v$. But $v \varrho_{\mathscr{L} \mathscr{\mathscr { L }}}$ is determined by $(\mathbf{s} v, \hat{\mathbf{s}} v)$ and $\mathbf{s} v \in \hat{\mathbf{s}} v$. Since $\hat{\mathbf{s}} v$ is finite, the $\mathscr{R}$-class of $v \varrho_{\mathscr{L} \mathscr{F}}$. is finite for any $v$. The mapping $x \mapsto x^{*}$ induces a bijection between $R_{x}$ and $L_{x} *$. Hence each $\mathscr{L}$-class of $F \mathscr{L} \mathscr{I}^{*}(X)$ is finite. But then each $\mathscr{D}$-class is finite and $F \mathscr{L} \mathscr{I}^{*}(X)$ is completely semisimple. A similar argument proves the ascending chain condition for $F \mathscr{L}^{*}(X) / \mathscr{R}$ respectively $F \mathscr{L} \mathscr{I}^{*}(X) / \mathscr{L}$.

\section{References}

[1] C. L. Adair: Bands with involution, J. Algebra 75 (1982), 297-314.

[2] $K$. Auinger: Bifree objects in $e$-varieties of strict orthodox semigroups and the lattice of strict orthodox *-semigroup varieties, Glasgow Math. J., to appear.

[3] K. Auinger: Strict regular *-semigroups, Proceedings of the 1991 Oberwolfach Semigroup Conference, to appear.

[4] A. H. Clifford: The free completely regular semigroup on a set, J. Algebra 59 (1979), 434-451.

[5] J. A. Gerhard and M. Petrich: Free bands and free *-bands, Glasgow Math. J. 28(1986), 161-179.

[6] J. A. Gerhard and M. Petrich: Free involutorial completely simple semigroups, Can. J. Math. 37 (1985), 271-295.

[7] T. E. Hall: Identities for existence varieties of regular semigroups, Bull. Austral. Math. Soc. 40 (1989), 59-77.

[8] T. E. Hall: Regular semigroups: amalgamation and the lattice of existence varieties, Algebra Universalis 28 (1991), 79-108.

[9] J. M. Howie: An Introduction to Semigroup Theory, Academic Press, London, 1976.

[10] H. Mitsch: A natural partial order for semigroups, Proc. Amer. Math. Soc. 97 (1986), 384-388.

[11] K. S. S. Nambooripad: The natural partial order on a regular semigroup, Proc. Edinburgh Math. Soc. 23 (1980), 249-260.

[12] K. S. S. Nambooripad and F. Pastijn: Regular involution semigroups, Semigroups, Coll. Math. Soc. János Bolyai 39 (1986), 199-249.

[13] T. E. Nordahl and H. E. Scheiblich: Regular *-semigroups, Semigroup Forum 16 (1978), 369-377.

[14] F. Pastijn: The structure of pseudo-inverse semigroups, Trans. Amer. Math. Soc. 27.3 (1982), 631-655.

[15] F. Pastijn: Rectangular bands of inverse semigroups, Simon Stevin 56 (1982), 1-97. 
[16] M. Petrich: Certain varieties of completely regular *-semigroups, Boll. Un. Mat. It. 4(B) (1985), 343-370.

[17] M. Petrich: Inverse Semigroups, Wiley, New York, 1984.

[18] L. Polák: Varieties of unary semigroups.

[19] B. Pondélícek: On varieties of regular *-semigroups, Czechoslovak Math. J. 41 (1991), 110-119.

[20] H. E. Scheiblich: Free inverse semigroups, Proc. Amer. Math. Soc. 38 (1973), 1-7.

[21] H. E. Scheiblich: Generalized inverse semigroups with involution, Rocky Mountain J. Math. 12 (1982), 205-211.

[22] B. M. Schein: Free inverse semigroups are not finitely presentable, Acta Math. Acad. Sci. Hung. 26 (1975), 41-52.

[23] M. B. Szendrei: Free *-orthodox semigroups, Simon Stevin 59 (1985), 175-201.

[24] M. B. Szendrei: A new interpretation of free orthodox and generalized inverse *-semigroups, Springer Lecture Notes 1320 (1986), 358-371.

Author's address: Institut für Mathematik, Strudlhofgasse 4, A-1090 Wien, Austria (email: a8131datQauniwi11.bitnet). 